\title{
Downregulation of RIP3 Improves the Protective Effect of ATF6 in an Acute Liver Injury Model
}

\author{
Mei-Ying Huang, ${ }^{1}$ Dian-Wei Wan, ${ }^{2}$ Jie Deng, ${ }^{2}$ Wen-Jie Guo, ${ }^{2}$ Yue Huang, ${ }^{2}$ Huan Chen, \\ De-Lin Xu, ${ }^{3}$ Zhi-Gang Jiang, ${ }^{4}$ Yuan Xue $\left(10,{ }^{5}\right.$ and Yi-Huai He ${ }^{2}{ }^{2}$ \\ ${ }^{1}$ Department of Pediatrics, The Affiliated Hospital of Zunyi Medical University, Zunyi, 563000 Guizhou, China \\ ${ }^{2}$ Department of Infectious Diseases, The Affiliated Hospital of Zunyi Medical University, Zunyi, 563000 Guizhou, China \\ ${ }^{3}$ Department of Cell Biology, Zunyi Medical University, Zunyi, 563099 Guizhou, China \\ ${ }^{4}$ School of Public Health, Zunyi Medical University, Zunyi, 563099 Guizhou, China \\ ${ }^{5}$ Department of Liver Diseases, The Third People's Hospital of Changzhou, Changzhou, 213000 Jiangsu Province, China
}

Correspondence should be addressed to Yi-Huai He; 993565989@qq.com

Received 23 July 2021; Revised 28 September 2021; Accepted 11 October 2021; Published 5 November 2021

Academic Editor: Dorota Formanowicz

Copyright (c) 2021 Mei-Ying Huang et al. This is an open access article distributed under the Creative Commons Attribution License, which permits unrestricted use, distribution, and reproduction in any medium, provided the original work is properly cited.

\begin{abstract}
Background. Activating transcription factor 6 (ATF6) and receptor-interacting protein 3 (RIP3) are important signaling proteins in endoplasmic reticulum (ER) stress and necroptosis, respectively. However, their regulatory relationship and clinical significance are unknown. We investigate the impact of ATF6 on RIP3 expression, and its role in hepatocyte necroptosis in an acute liver injury model. Methods. In vivo and in vitro experiments were carried out. LO2 cells were treated with thapsigargin (TG). In vivo, male $\mathrm{BALB} / \mathrm{c}$ mice were treated with carbon tetrachloride $\left(\mathrm{CCl}_{4}, 1 \mathrm{~mL} / \mathrm{kg}\right)$ or tunicamycin $(\mathrm{TM}, 2 \mathrm{mg} / \mathrm{kg})$. Then, the impact of ATF6 or RIP3 silencing on liver injury, hepatocyte necroptosis, and ER stress-related protein expression was examined. Results. TG induced ER stress and necroptosis and ATF6 and RIP3 expression in LO2 cells. The knockdown of ATF6 significantly decreased RIP3 expression $(p<0.05)$ and increased ER stress and necroptosis. The downregulation of RIP3 significantly reduced necroptosis and ER stress $(p<0.05)$. Similar results were observed in $\mathrm{CCl}_{4}$ or the TM-induced mouse model. The knockdown of ATF6 significantly decreased $\mathrm{CCl}_{4}$-induced RIP3 expression and increased liver injury, necroptosis, and ER stress in mice livers $(p<0.05)$. In contrast, the downregulation of RIP3 significantly reduced liver injury, hepatocyte necroptosis, and ER stress. Conclusions. Hepatocyte ATF6 has multiple roles in acute liver injury. It reduces hepatocyte necroptosis via negative feedback regulation of ER stress. In addition, ATF6 can upregulate the expression of RIP3, which is not helpful to the recovery process. However, downregulating RIP3 reduces hepatocyte necroptosis by promoting the alleviation of ER stress. The findings suggest that RIP3 could be a plausible target for the treatment of liver injury.
\end{abstract}

\section{Introduction}

Hepatocyte necroptosis is considered to be a crucial pathologic pathway involved in several diseases [1]. Hepatocyte necroptosis has been associated with the development of pathologic liver conditions $[2,3]$. Necroptosis is a caspase-independent mode of cell death that is regulated via multiple proteins that include receptor-interacting protein kinase-1 (RIP1), receptor-interacting protein kinase-3 (RIP3), and mixed lineage kinase domain-like protein (MLKL) [4]. RIP1 and RIP3 bind together forming a RIP1-RIP3 complex. Then, this complex recruits other molecules including the tumor necrosis factor receptor type 1-associated death domain (TRADD), Fas, and tumor necrosis factor receptor-1 (TNFRI) and activates MLKL. MLKL is the terminal mediator for necroptosis [5].

The necroptotic complex that is formed by RIP1, RIP3, and MLKL is crucial for necroptosis [6]. RIP3 binds to the MLKL C-terminus, which stimulates the phosphorylation of the Thr357/Ser358 sites and promotes the activation of the necroptotic complex [7]. Subsequently, activated MLKL 
TABLE 1: List of shRNA sequences.

\begin{tabular}{|c|c|c|c|}
\hline \multicolumn{3}{|c|}{ Insert content } & $5^{\prime}$ to $3^{\prime}$ \\
\hline \multirow{6}{*}{ Human } & \multirow{3}{*}{ ATF6 } & Target sequence & GCAGGTCCTCCTGTTATTAGA \\
\hline & & ATF6 shRNA & GCAGGTCCTCCTGTTATTAGACGAATCTAATAACAGGAGGACCTGC \\
\hline & & Control shRNA & AAACGTGACACGTTCGGAGAACGAATTCTCCGAACGTGTCACGTTT \\
\hline & \multirow{3}{*}{ RIP3 } & Target sequence & GCAAGTCTGGATAACGAATTC \\
\hline & & RIP3 shRNA & GCAAGTCTGGATAACGAATTCCGAAGAATTCGTTATCCAGACTTGC \\
\hline & & Control shRNA & AAACGTGACACGTTCGGAGAA \\
\hline \multirow{6}{*}{ Mouse } & \multirow{3}{*}{ Atf6 } & Target sequence & GCAGTCGATTATCAGCATACA \\
\hline & & Atf6 shRNA & GCAGTCGATTATCAGCATACACGAATGTATGCTGATAATCGACTGC \\
\hline & & Control shRNA & AAACGTGACACGTTCGGAGAACGAATTCTCCGAACGTGTCACGTTT \\
\hline & \multirow{3}{*}{ Rip3 } & Target sequence & GGACCCAGAGCTGTTATTTGA \\
\hline & & Rip3 shRNA & GGACCCAGAGCTGTTATTTGACGAATCAAATAACAGCTCTGGGTCC \\
\hline & & Control shRNA & AAACGTGACACGTTCGGAGAACGAATTCTCCGAACGTGTCACGTTT \\
\hline
\end{tabular}

migrates to the plasma membrane where it binds to phospholipid-dylinositol lipids via its $\mathrm{N}$-terminal region, causing ion influx through the ion channels and leading to the swelling of organelles, and cell death [8]. Downregulation or silencing MLKL inhibits necroptosis [9]. Therefore, compounds that downregulate MLKL or inhibit its phosphorylation could be promising therapeutic agents. For instance, Necrostatin-1, a RIP1 inhibitor, inhibits necroptosis [10].

Endoplasmic reticulum (ER) stress regulates necroptosis, which is involved in several pathologic conditions [11, 12]. ER is important for protein synthesis, folding, and secretion in eukaryotic cells. Cells have evolved a highly regulated mechanism to maintain homeostasis, which helps during folding and the modification of proteins in ER. With the accumulation of misfolded proteins, eukaryotic cells enhance their proteinfolding ability, arrest protein translation, and accelerate protein degradation. With the failure to regain intracellular homeostasis, the cells will activate C/ERB homologous transcription factor protein (CHOP), c-Jun $\mathrm{N}$-terminal kinase (JNK), and/or caspase-12 signaling, which initiates a damaging response $[13,14]$. In combination, these responses are referred to as an unfolded protein response (UPR) [15]. The UPR is mediated by the inositol-requiring enzyme 1 (IRE1), activating transcription factor 6 (ATF6), and protein kinase R-like endoplasmic reticulum kinase (PERK) [16]. Under physiological conditions, glucose-regulated protein 78 (GRP78) binds to IRE1, PERK, and ATF6, and it inhibits their activation. During ER stress, unfolded or misfolded proteins increase and compete with GRP78. Following dissociation from GRP78, IRE1, PERK, and ATF6 are activated, respectively [17]. ATF6 (P90ATF6) dissociates from GRP78 and transfers to the Golgi apparatus, where it is hydrolyzed into an active fragment (P50ATF6).

ER stress regulates necroptosis via the tumor necrosis factor-alpha- (TNF- $\alpha$-), caspase-8-, RIP1-, RIP3-, MIKL-, and NF- $\kappa \mathrm{B}-$-mediated signaling [18]. Caspase- 8 mediates stress-induced necroptosis independent of apoptotic signaling during ER stress [19]. Tauroursodeoxycholic acid hampers ER stress, downregulates RIP1 and RIP3 expression, and reduces necroptosis [20]. IRE1 activates NF- $\kappa$ B, causes
TNF- $\alpha$ secretion, and mediates necroptosis through tumor necrosis factor receptor 1 (TNFR1) [21, 22]. Regulation of the JNK signaling pathway can affect necroptosis [23, 24]. However, the effects of ATF6 on the necroptotic signaling pathway in acute liver injury remain unknown. ATF6mediated signaling upregulates molecule chaperones, activates the transcription of ER stress genes, and improves protein folding [25-27]. Previous studies demonstrated the activation of RIP3 by the ER stress signaling pathway [28, 29]. ER stress mediates necroptosis [30]. In this study, the impact of ATF6 on necroptotic signaling in acute liver injury in vitro and in vivo will be investigated.

\section{Material and Methods}

2.1. Induction of ER Stress In Vitro. LO2 cells were purchased from the Cell Bank of the Type Culture Collection of the Chinese Academy of Sciences (Shanghai, China) and maintained in RPMI-1640 with $10 \%$ fetal bovine serum and $1 \%$ penicillin/streptomycin. LO2 cells are adherent immortalized human normal hepatocytes with typical morphological characteristics of hepatocytes. They are widely used in experimental studies into liver diseases. To activate ER stress, thapsigargin (TG) was used to treat $\mathrm{LO} 2$ cells. TG is an ER membrane $\mathrm{Ca}^{2+}$-ATPase that disturbs calcium homeostasis in ER and reduces protein-folding capacity that ultimately induces ER stress in vitro [31, 32]. LO2 cells were incubated with dimethyl sulfoxide (DMSO; control group) or $0.5 \mu \mathrm{mol} / \mathrm{L}$ TG (Sigma-Aldrich, USA) for $12,24,48$, or $72 \mathrm{~h}$. In addition, $1.0 \times 10^{6} \mathrm{LO} 2$ cells were seeded in a 6 well plate, and the target or control short hairpin RNA (shRNA; Syngen Tech Co., Ltd., Beijing, China) were introduced via lentiviral vectors for $48 \mathrm{~h}$ (Table 1 ) and then cells were incubated with TG for $24 \mathrm{~h}$ to induce ER stress.

2.2. Generation of an Acute Liver Injury Mouse Model. Male $\mathrm{BALB} / \mathrm{c}$ mice $(25 \pm 3 \mathrm{~g}$; Animal Center of Zunyi Medical University, Guizhou, China) were maintained under specific pathogen-free conditions ( $12 \mathrm{~h}$ light/dark cycle with food and water available ad libitum). A total of 240 animals were 
used in this study, and the research protocol was approved by the Animal Ethics Committee of the Affiliated Hospital of Zunyi Medical University (Guizhou Province, China; NO: KLLY(A)-2019-095) in agreement with the Animal Care and Research guidelines [33].

Following 1 week of acclimatization, mice were randomly assigned to model groups $\left(\mathrm{CCl}_{4}\right.$ or $\left.\mathrm{TM}\right)$ and solvent control groups (olive oil for $\mathrm{CCl}_{4}$ or phosphate-buffered saline (PBS) as the TM solvent control). All treatments were administered via the intraperitoneal route, and experimental outcomes were detected at 12,24 , and $48 \mathrm{~h}$ postinjection as detailed previously $[30,34]$. In the $\mathrm{CCl}_{4}$ group, mice received $1 \mathrm{~mL} / \mathrm{kg} \mathrm{CCl}_{4}$, and mice in the olive oil control group received $4 \mathrm{~mL} / \mathrm{kg}$ olive oil. In the TM group, mice received $2 \mathrm{mg} / \mathrm{kg} \mathrm{TM}$, and the control group received $5 \mathrm{~mL} / \mathrm{kg}$ PBS. Alanine aminotransferase (ALT), total bilirubin (TBil), and the area of necrotic liver tissue were used to assess liver injury.

To examine the impact of AFT6 or RIP3 on ER stressmediated apoptosis, mice were administered recombinant adenovirus-associated vector serotype 8 (rAAV8; $2 \times 10^{10}$ viral genome copies in $100 \mu \mathrm{L}$ PBS) that expressed Atf6 or Rip3 short hairpin RNA or control shRNA (Syngen Tech Co., Ltd., Beijing, China; Table 1) via the tail vein as described previously [35]. In the target shRNA or control shRNA groups, $\mathrm{CCl}_{4}$ was administered 6 weeks after rAAV8 transduction $(n=12)$. At the end of each experiment, mice were sacrificed by $\mathrm{CO}_{2}$ euthanasia, and tissue and blood samples were obtained [36].

2.3. Analysis of Protein Expression. Total proteins were obtained from liver tissues using immunoprecipitation lysis buffer (R0010, Solarbio, Beijing, China). In total, $10 \mathrm{mg}$ of liver tissues was homogenized in the protein lysis buffer $(1 \mathrm{~mL})$ that was supplemented with $10 \mu \mathrm{L}$ PMSF, using a glass homogenization tube at $4^{\circ} \mathrm{C}$. The homogenates were further disturbed using an ultrasonic cell disruptor (Jingxin Industrial Development Co., Ltd., Shanghai, China). Adherent cells were lysed with protein lysis buffer that contained PMSF (0.25-0.5 mL/well according to the cell density) at $4{ }^{\circ} \mathrm{C}$. Then, lysates were cleared by centrifugation at $14000 \mathrm{~g}$, at $4^{\circ} \mathrm{C}$ for $15 \mathrm{~min}$ (Catcher Instrument Co., Ltd., Hunan, China). The protein concentration was detected in the supernatant using the bicinchoninic acid (BCA) method according to standard protocols [37]. In total, $40 \mu \mathrm{g}$ protein extracts were resolved on 10\% SDS-PAGE by a vertical electrophoresis transfer system (Bio-Rad, Hercules, CA, USA) and transferred to polyvinylidene fluoride membranes (Millipore, Billerica, MA, USA). Following blocking, membranes were incubated with mouse monoclonal antibodies against ATF6 (sc-166659, 1:1000, Cell Signaling Technology, USA), CHOP (GADD153, sc-71136, 1:10000, Santa Cruz Biotechnology, USA), glyceraldehyde 3-phosphate dehydrogenase (GAPDH; sc-365062, 1:1000, Santa Cruz Biotechnology), RIP3 (sc-374639, $1: 1000$, Santa Cruz Biotechnology, USA), rabbit monoclonal antibodies, phosphorylated MLKL (p-MLKL, 37333S, 1:1000, Cell Signaling Technology, USA), MLKL (PA5-34733, 1:1000, Thermo Fisher Scientific, USA), or rabbit polyclonal antibodies against caspase-12 (2202, 1:1000, Cell Signaling Technology). Protein bands were detected with enhanced chemiluminescent reagents (E002-100, 7Sea Pharmtech Co., Ltd., Shanghai, China), and the results were recorded using a chemiluminescence imaging system (Clinx, ChemiScope 6000, Shanghai, China). Densitometric analysis was used to determine the intensity of protein bands, and the data was processed with Quantity One software (Bio-Rad, Hercules, CA, USA).

2.4. Cell Viability Assay. The Cell Titer 96 Aqueous One Solution Cell Proliferation assay kit (Cat. No. 40203ES60; Yeasen; Shanghai Yi San Biotechnology Co., Ltd., Shanghai, China) was used to detect relative LO2 cell viability. The cell suspension ( $100 \mu \mathrm{L} /$ well containing approximately 5,000 LO2 cells) was seeded into a 96-well plate ( 5 replicates per sample). The cell culture plates were placed in the incubator for preculture $\left(37^{\circ} \mathrm{C}, 5 \% \mathrm{CO}_{2}\right)$. When $60 \%-90 \%$ cell density was reached, the LO 2 cells were subjected to different experimental conditions. At the end of each experiment, the cell culture media was aspirated and $100 \mu \mathrm{L}$ of the diluted CCK8 reagent was added (diluted with serum-free cell culture solution at a ratio of 1:9). The 96-well plate was incubated for $1 \mathrm{~h}$, and the absorbance was recorded at $450 \mathrm{~nm}$ on a microplate reader (Bio-Rad, CA, United States). To determine cell viability, we used the following:

$$
\text { Cell viability } \times 100 \% \text {. }
$$

2.5. Pathological Analysis of Liver Tissue. Fresh liver tissue $(5 \mathrm{~mm} \times 5 \mathrm{~mm})$ was fixed in $4 \%$ paraformaldehyde for $\geq 24 \mathrm{~h}$ and dehydrated in a gradient alcohol series, embedded in paraffin, and cut into $5 \mu \mathrm{m}$ thick liver sections. The paraffin sections were dewaxed and rehydrated and stained with hematoxylin and eosin for the nucleus and cytoplasm, respectively. Sections were then dehydrated and mounted on slides; then, the slides were scanned on a sliced panoramic scanner (Pannoramic DESK/MIDI/250/1000, 3DHISTECH, Hungary), observed, and photographed using CaseViewer 2.4 software (3DHISTECH, Hungary). Finally, the area of liver necrosis was analyzed by Image-Pro Plus 6.0 (Media Cybernetics, USA) [30].

2.6. Assessment of Liver Function. Serum levels of ALT and TBil were detected using the rate and diazonium (Beckman Coulter autoanalyzer, AU5800, USA) methods, as detailed previously [38].

2.7. Statistical Analysis. Data were represented as means \pm standard deviation. Differences between the different experimental groups were estimated using one-way analysis of variance with Tukey's post hoc analysis (ANOVA), and the least significant difference (LSD) test was used for pairwise comparison. A $p$ value of $<0.05$ was regarded statistically significant.

\section{Results}

3.1. TG Stimulates ER Stress and Necroptosis and Upregulation of RIP3 Expression in LO2 Cells. Compared 


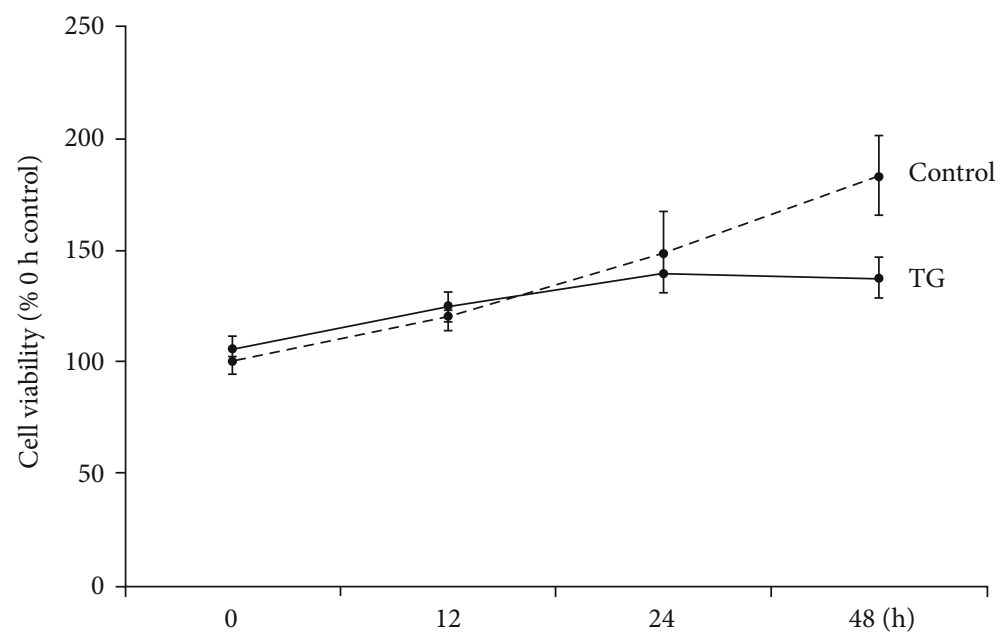

(a)
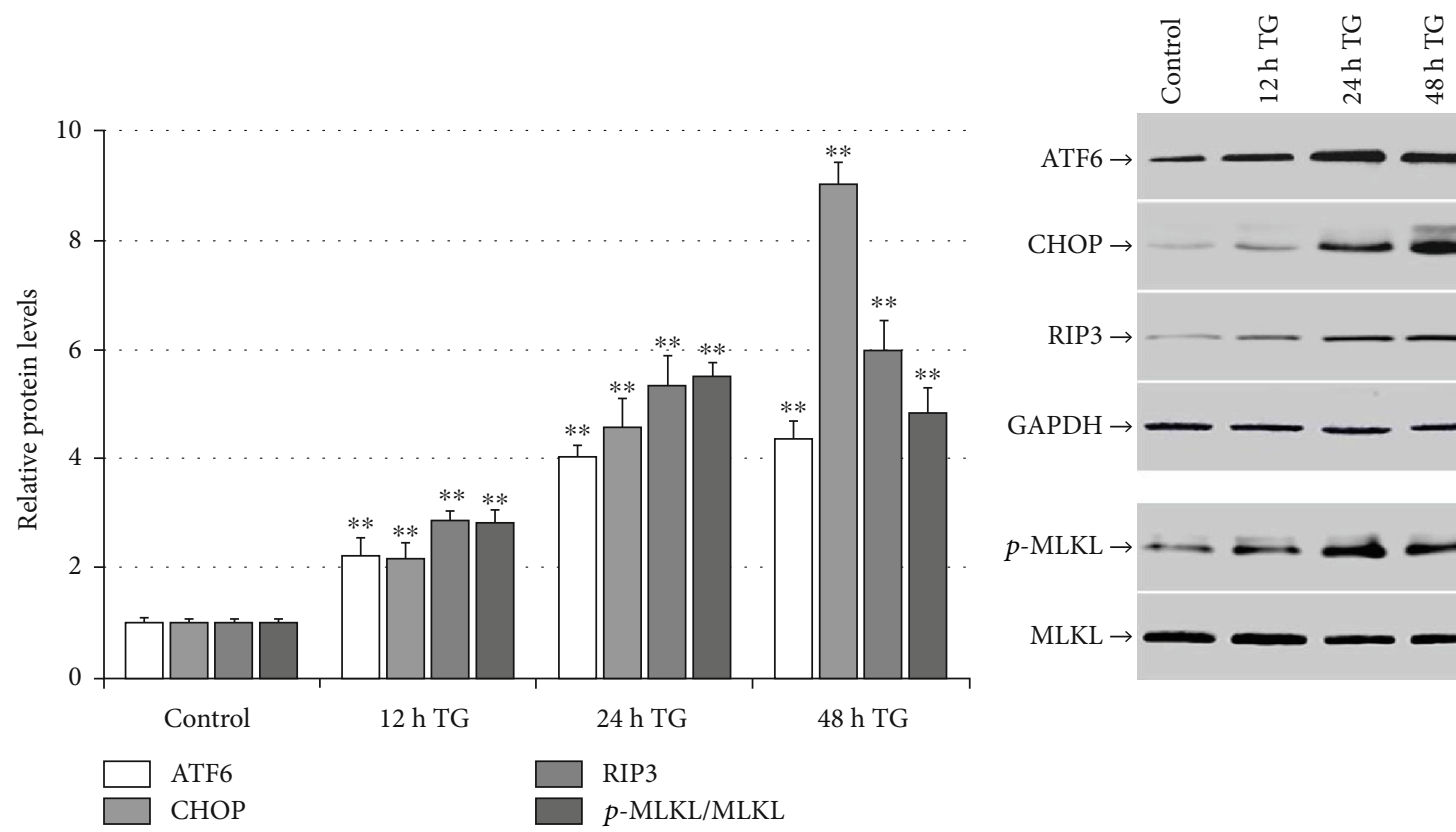

(b)

FIGURE 1: TG promotes ER stress and necroptosis and upregulates RIP3 expression in LO2 cells. LO2 cells were treated with DMSO (control group) or TG $(0.5 \mu \mathrm{mol} / \mathrm{L})$ for 12,24 , or $48 \mathrm{~h}$ : (a) time-dependent cell viability was detected by CCK8 assay following TG treatment, and (b) time-dependent related protein expression was detected by western blotting following TG treatment. ${ }^{*} p<0.05$ and ${ }^{* *} p<0.01$ versus the control group.

with the DMSO-treated (control) cells, TG treatment significantly reduced LO2 cell viability at 24 and $48 \mathrm{~h}(p<0.05$; Figure 1(a)). In addition, TG treatment upregulated the protein levels of ATF6, CHOP, RIP3, and p-MLKL at 12, 24, and $48 \mathrm{~h}(p<0.01$; Figure 1(b)).

3.2. ATF6 Knockdown Aggravates TG-Induced Necroptosis and ER Stress and Reduced RIP3 Expression in LO2 Cells. Compared with the control shRNA, ATF6 shRNA reduced LO2 viability $(p<0.05$; Figure $2(\mathrm{a}))$, and it decreased ATF6 and RIP3 expression; however, it increased p-MLKL expression $(p<0.05$; Figure 2(b)). Following TG treatment, ATF6 shRNA further decreased LO2 viability and RIP3 expression, but it elevated p-MLKL levels compared with the control shRNA-TG group (control shRNA + TG; $p<0.05$; Figures 2(a) and 2(b)). Compared with the control shRNATG group, ATF6 shRNA-TG induction increased the expression of CHOP protein $(p<0.01$; Figure $2(c))$.

3.3. RIP3 Downregulation Alleviates TG-Induced Necroptosis and ER Stress in LO2 Cells. Compared with the control shRNA, RIP3 shRNA increased LO2 viability but decreased RIP3 and p-MLKL expression ( $p<0.01$; Figure 3(a)). Transfection with RIP3 shRNA and TG treatment (RIP3 shRNATG group) increased LO2 cell viability but reduced RIP3 and p-MLKL levels compared with the control shRNA-TG group 


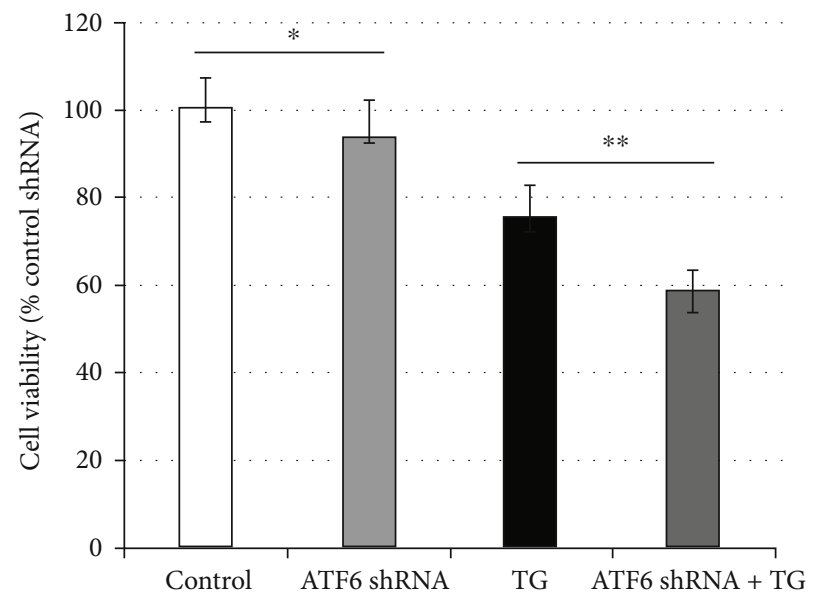

(a)
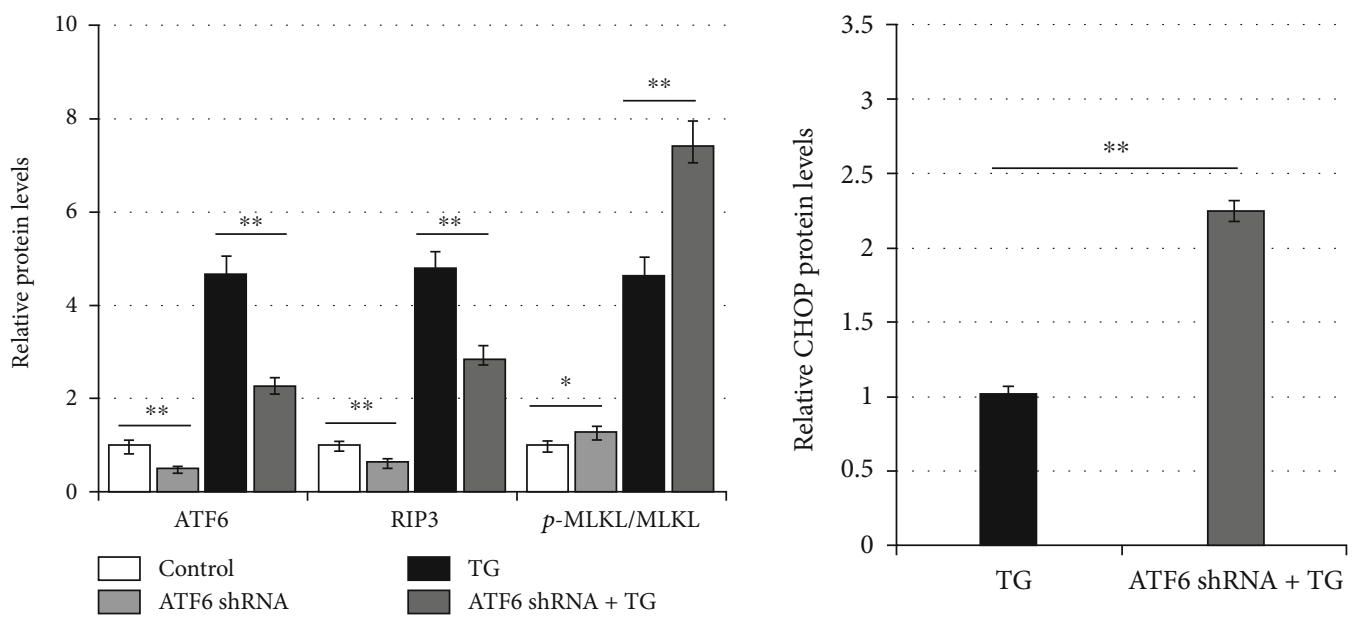

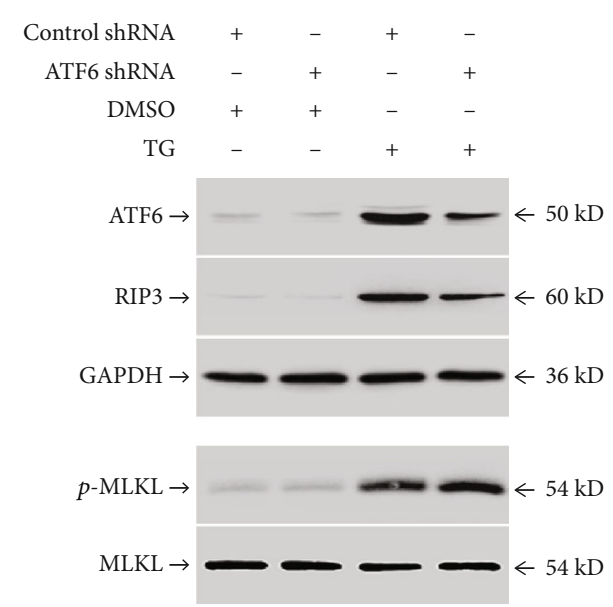

(b)

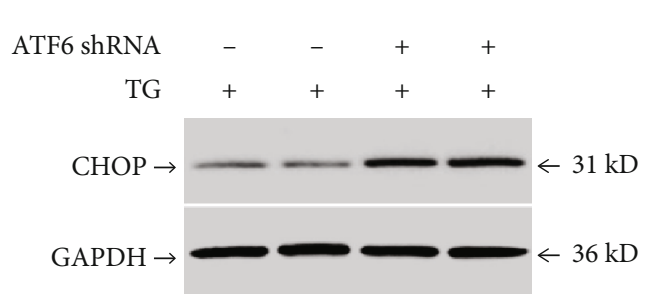

(c)

FIGURE 2: ATF6 silencing aggravates TG-induced necroptosis and ER stress and reduces RIP3 expression in LO2 cells. LO2 cells were infected with control shRNA or ATF6 shRNA for $48 \mathrm{~h}$, and then they were incubated with DMSO or TG (0.5 $\mu \mathrm{mol} / \mathrm{L})$ for another $24 \mathrm{~h}$ : (a) comparison of cell viability between the control group (control shRNA + DMSO), the ATF6 shRNA group (ATF6 shRNA + DMSO), the TG group (control shRNA + TG), and the ATF6 shRNA + TG group in LO2 cells; (b) bar chart representing the ATF6, RIP3, and p-MLKL protein expression and representative western blotting analyzing the protein expression among the different experimental groups; (c) bar chart representing $\mathrm{CHOP}$ protein expression and representative western blotting evaluating the protein expression among the TG group and the ATF6 shRNA + TG group. ${ }^{*} p<0.05$ and ${ }^{* *} p<0.01$ versus the control group or the TG group. 


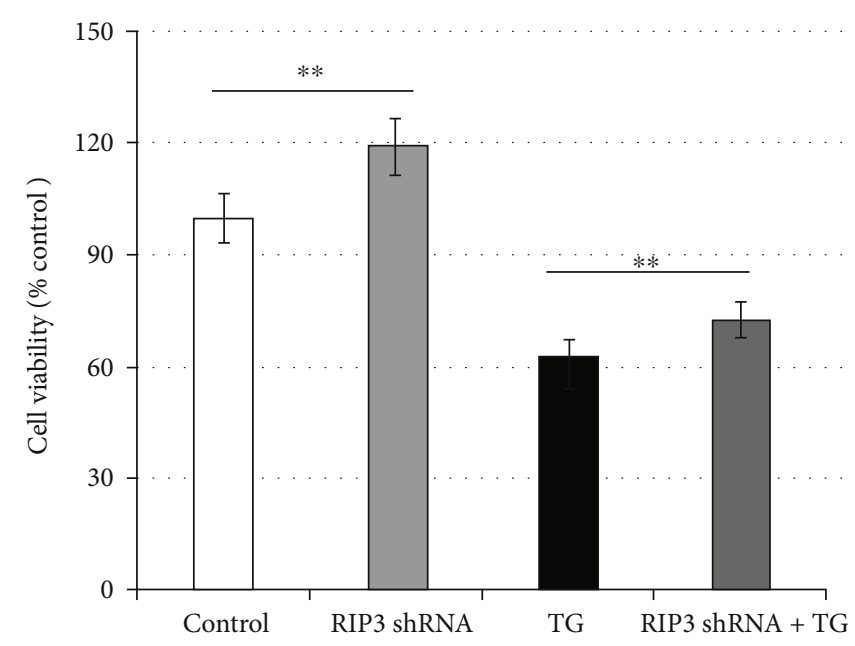

(a)

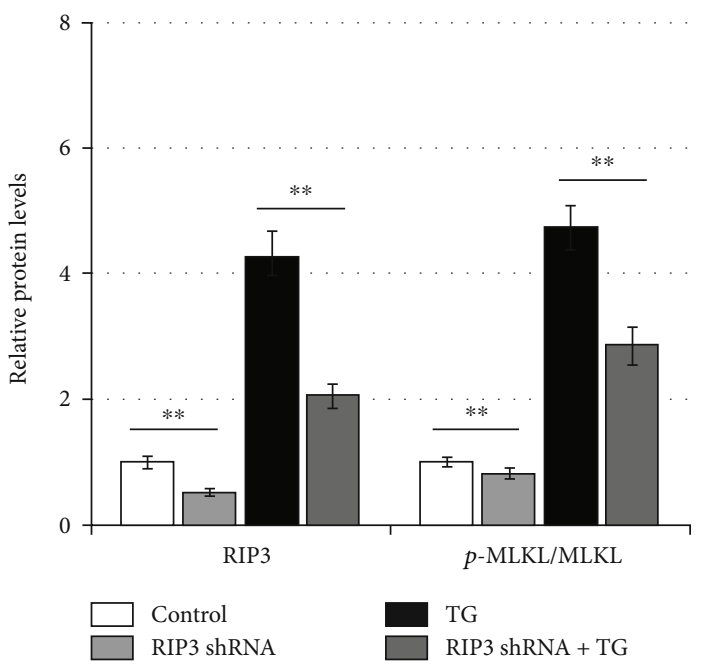

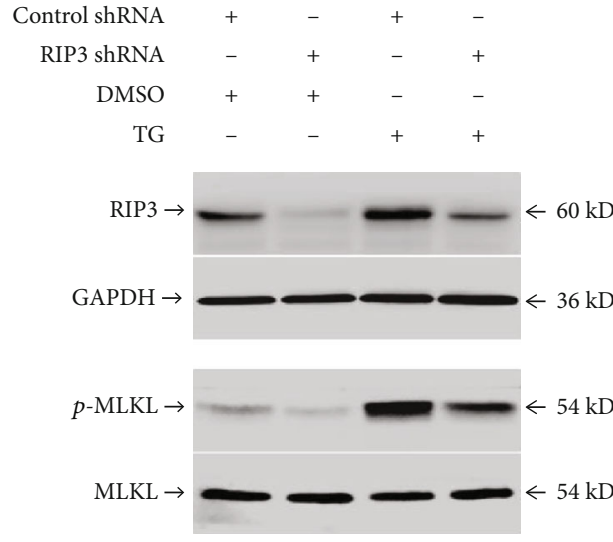

(b)
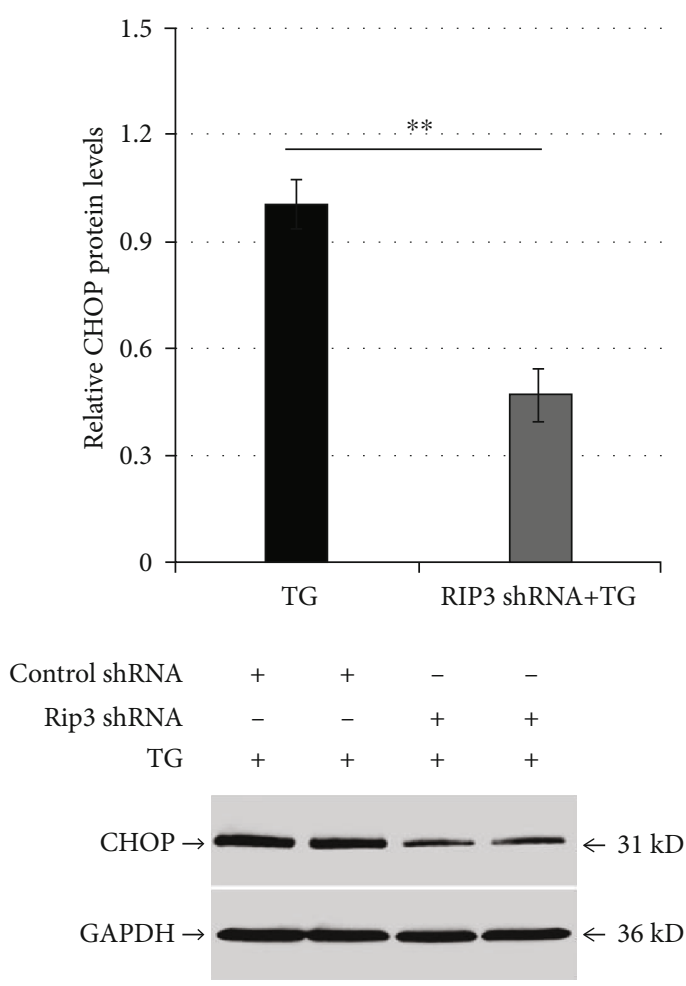

FIGURE 3: RIP3 silencing alleviates TG-induced necroptosis and ER stress in LO2 cells. LO2 cells were infected with control shRNA or RIP3 shRNA for $48 \mathrm{~h}$, and then they were incubated with DMSO or TG $(0.5 \mu \mathrm{mol} / \mathrm{L})$ for another $24 \mathrm{~h}$ : (a) comparison of cell viability between the control group (control shRNA + DMSO), the RIP3 shRNA group (RIP3 shRNA + DMSO), the TG group (control shRNA + TG), and the RIP3 shRNA + TG group in LO2 cells; (b) bar chart representing the RIP3 and p-MLKL protein expression and representative western blotting analyzing the protein expression among the different experimental groups; (c) bar chart representing CHOP protein expression and representative western blotting demonstrating the protein expression among the TG group and the ATF6 shRNA + TG group. ${ }^{* *} p$ $<0.01$ versus the control group or the TG group. 


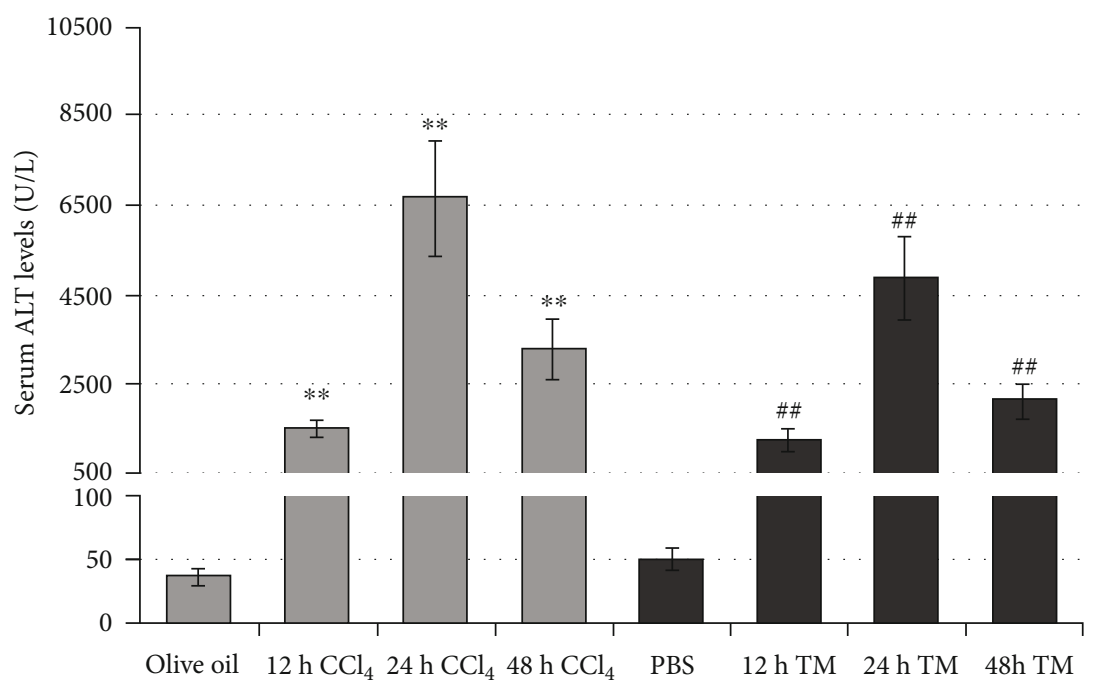

(a)

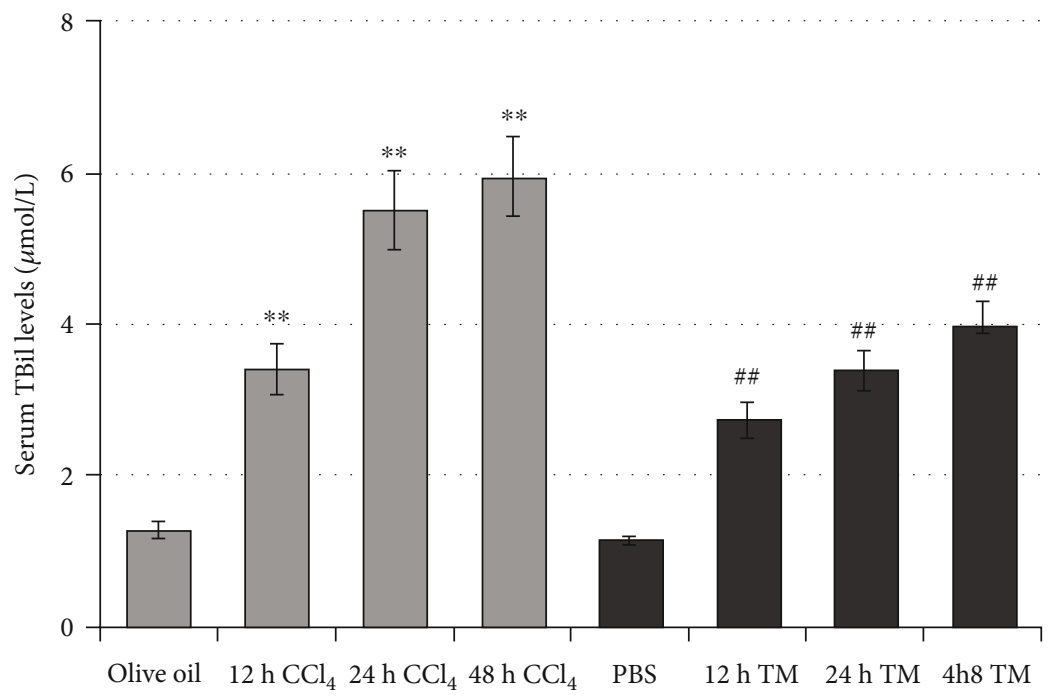

(b)

FIgURE 4: Continued. 

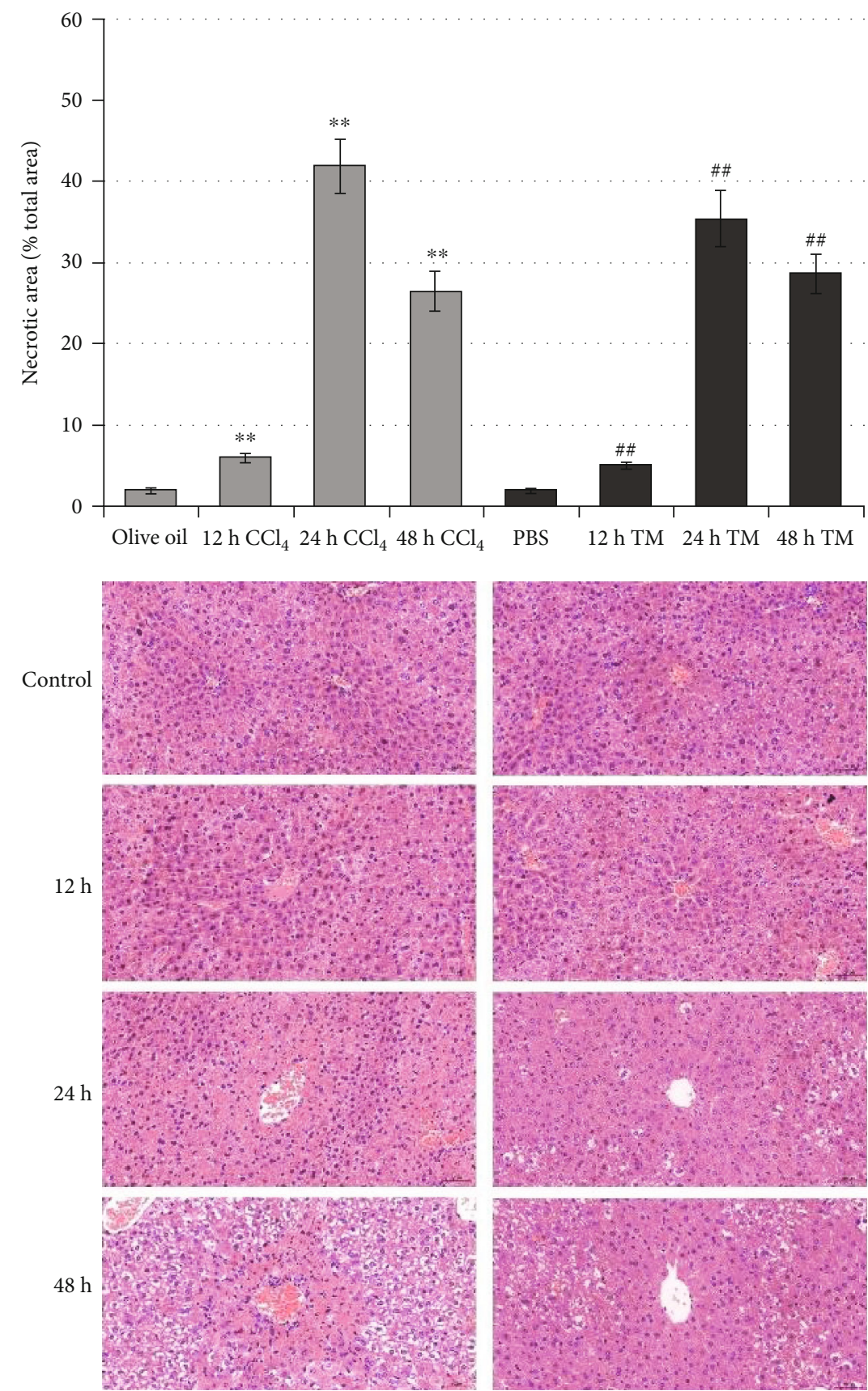

$\mathrm{CCl}_{4}(\mathrm{H} \& \mathrm{E}, 20 \times)$

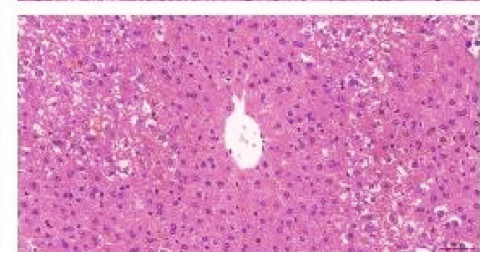

TM (H\&E, 20×)

(c)

Figure 4: Continued. 

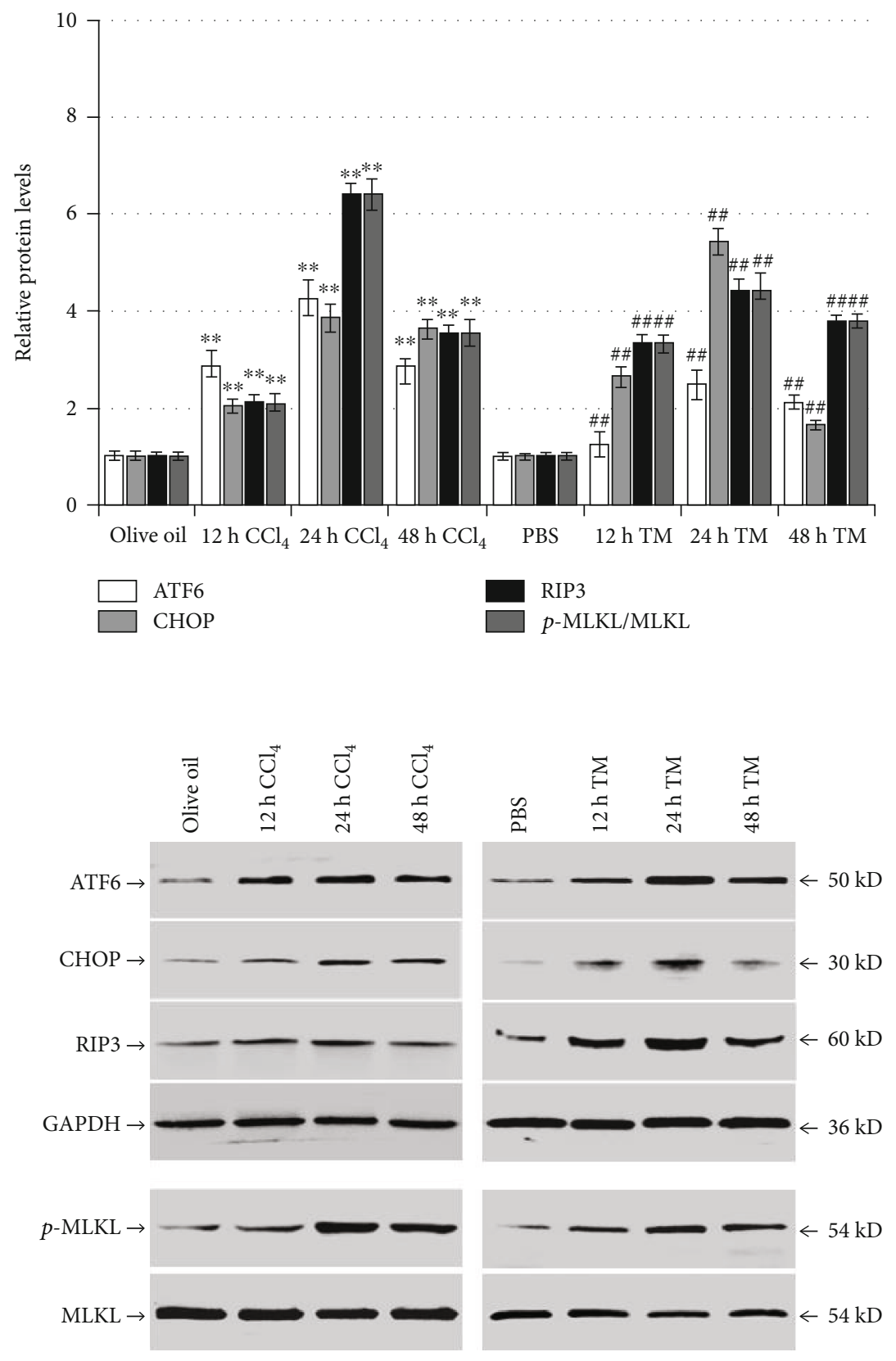

(d)

FIgURE 4: Induction of acute liver injury in mice. Male BALB/c mice were administered olive oil ( $\mathrm{CCl}_{4}$ solvent), $\mathrm{PBS}$ (TM solvent), CCl ${ }_{4}$, or

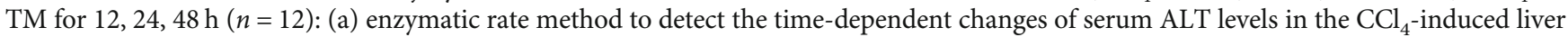
injury mouse model; (b) serum TBil levels measured using the diazonium method in the different experimental groups; (c) H\&E staining representing pathological changes in liver tissue and bar charts representing the proportion of necrotic liver tissue area; (d) protein expression of intrahepatic RIP3, CHOP, ATF6, and p-MLKL measured by western blotting after $\mathrm{CCl}_{4}$ injection. ${ }^{* *} p<0.01$ versus the olive oil group and ${ }^{\# \#} p<0.01$ versus the PBS group.

$(p<0.01$; Figure 3(b)). Finally, RIP3 shRNA significantly decreased CHOP protein expression in TG-induced cells $(p<0.01$; Figure 3(c)).

3.4. Induction of Acute Liver Injury in Mice. Acute liver injury was induced in mice by $\mathrm{CCl}_{4}$ or TM injection. Compared with the corresponding control solvent group, $\mathrm{CCl}_{4}$ and TM significantly increased serum ALT $(p<0.01$; Figure 4(a)), TBil levels $(p<0.01$; Figure $4(\mathrm{~b}))$, and the necrotic liver area $(p<0.01$; Figure $4(\mathrm{c}))$ at 12,24 , and $48 \mathrm{~h}$ after induction, which suggested the successful induction of acute liver injury. Therefore, the expression of ATF6, CHOP, RIP3, and p-MLKL proteins were significantly increased in the liver, which indicated the induction of necroptosis and ER stress along with acute liver injury $(p<0.01$; Figure $4(d))$.

3.5. ATF6 Silencing Aggravates Liver Injury and ER Stress and Reduces RIP3 Expression in $\mathrm{CCl}_{4}$-Induced Mice. Male 


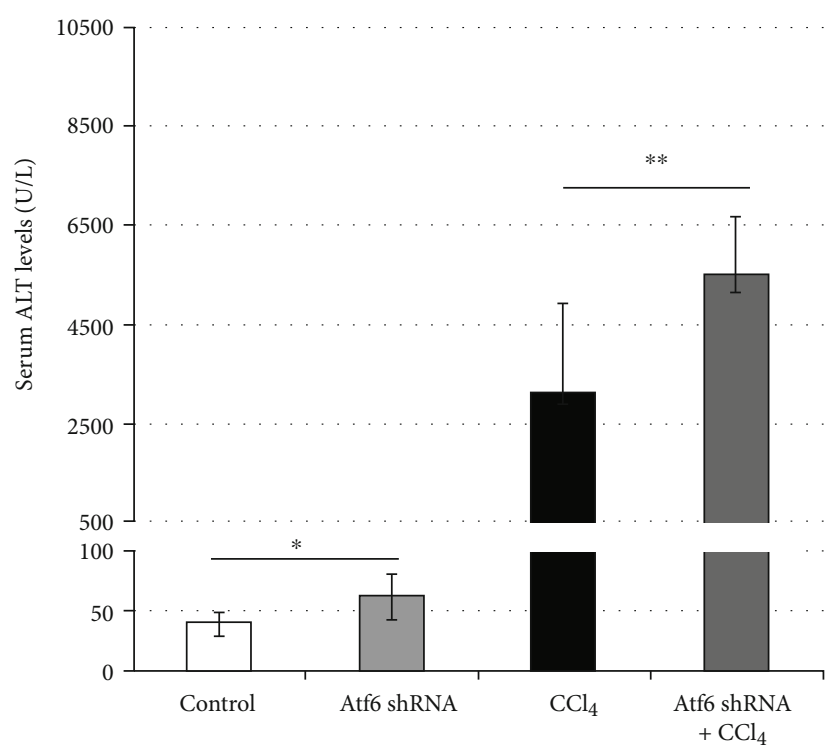

(a)

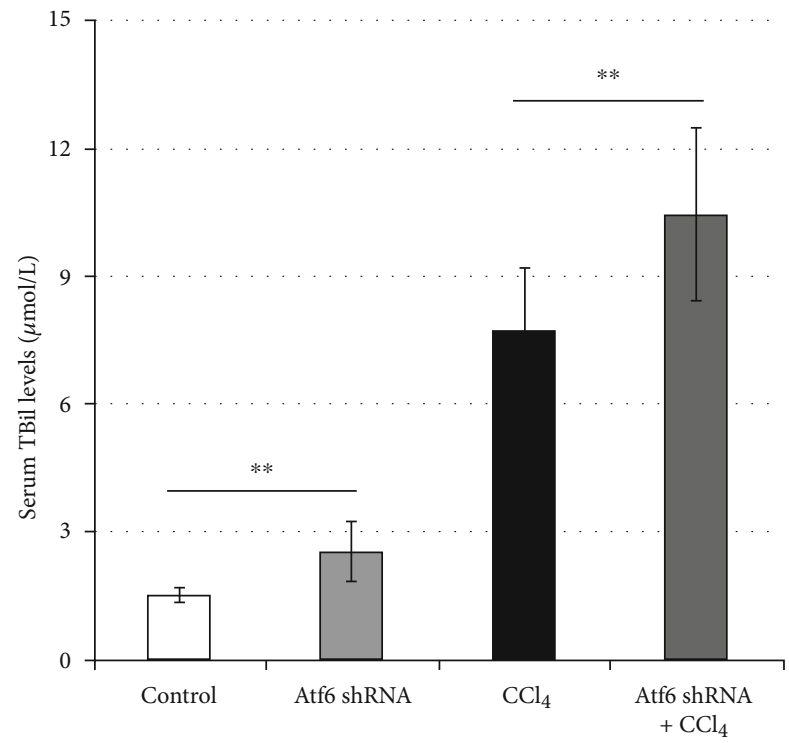

(b)
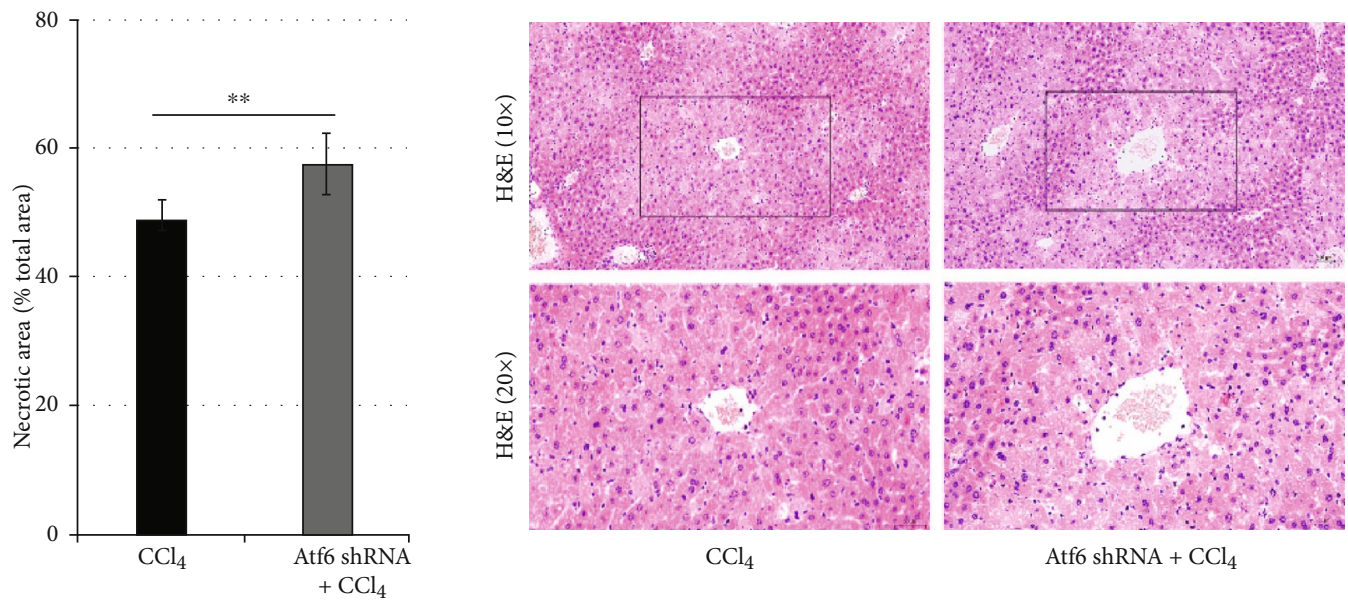

(c)

FIgURe 5: Continued. 


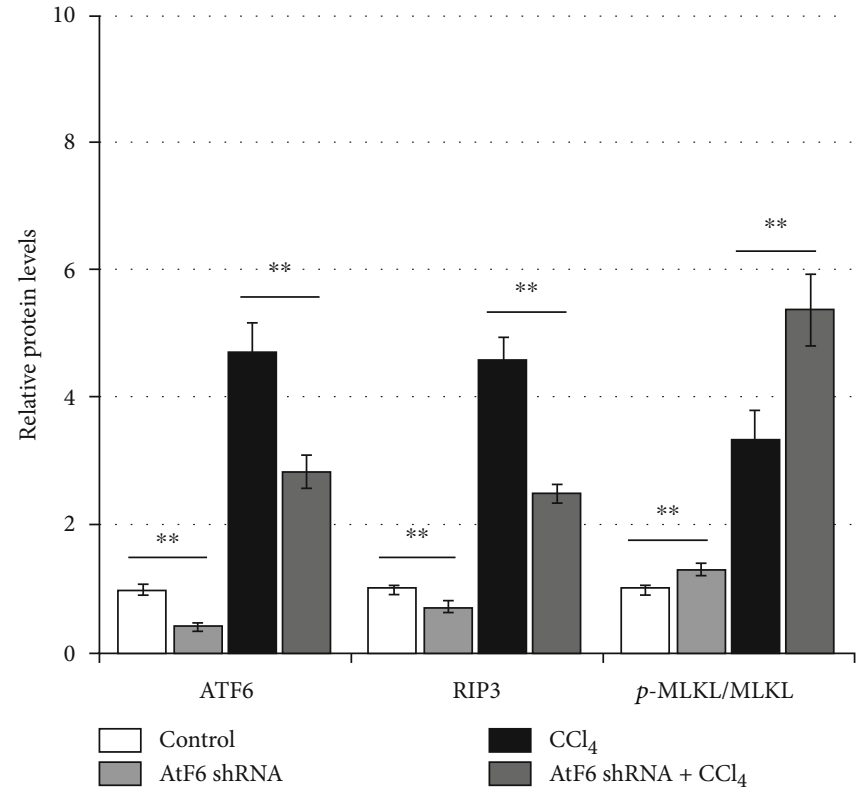

AtF6 shRNA $\square$ AtF6 shRNA + $\mathrm{CCl}_{4}$

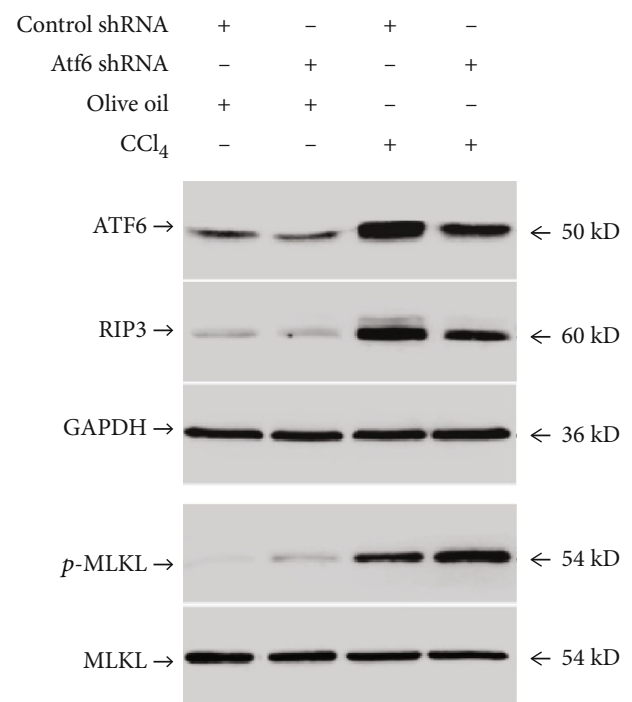

(d)
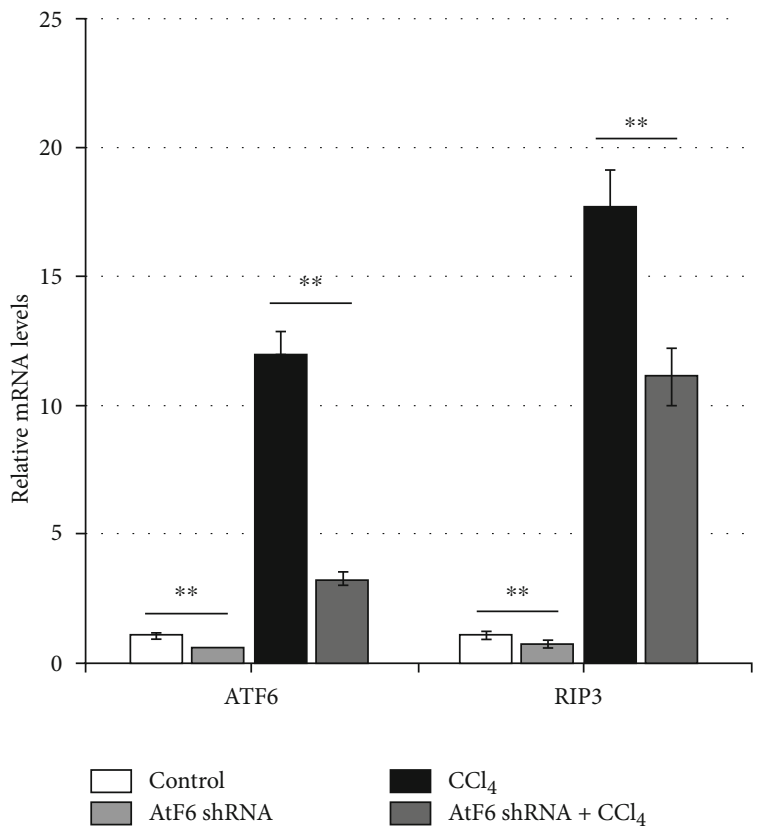

(e)

Figure 5: Continued. 


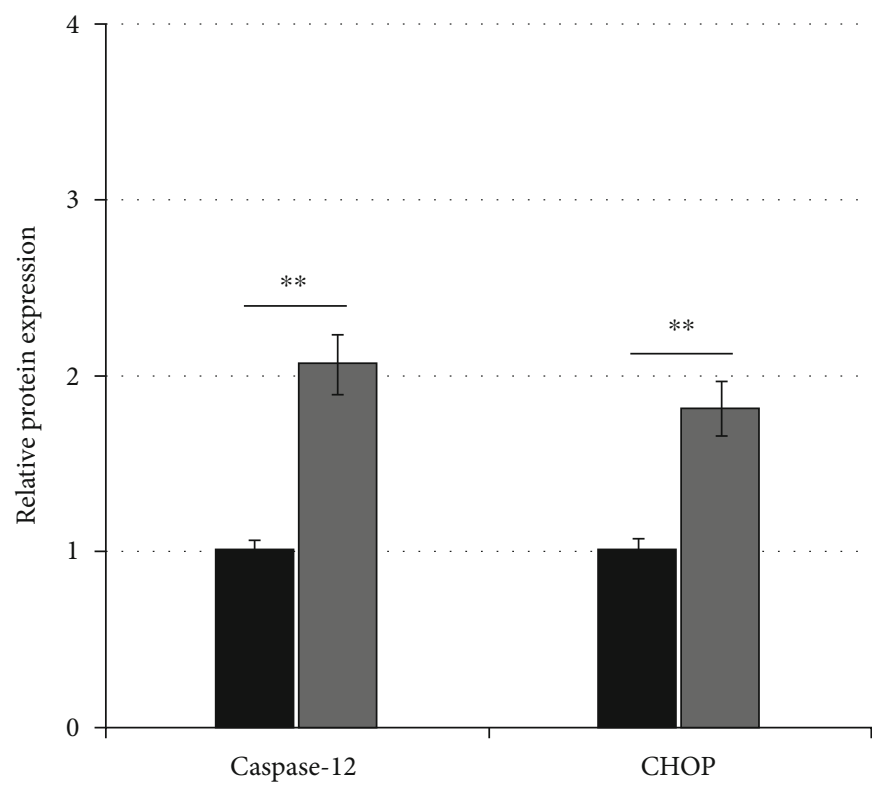

$\mathrm{CCl}_{4}$

AtF6 shRNA $+\mathrm{CCl}_{4}$

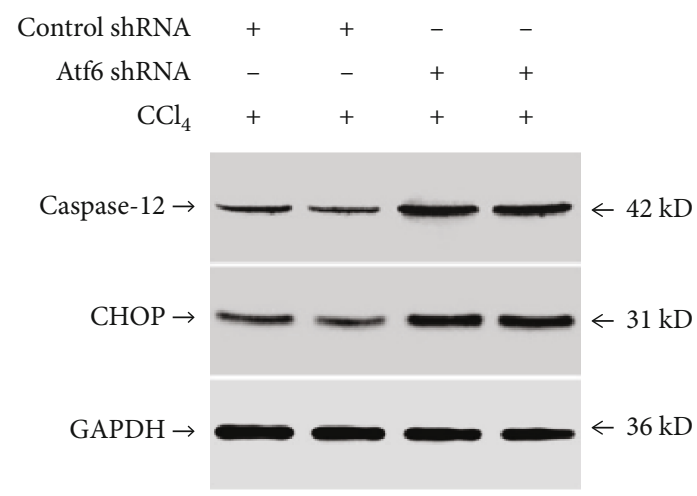

(f)

FIgURE 5: Atf6 knockdown aggravates liver injury and ER stress and reduces RIP3 expression in $\mathrm{CCl}_{4}$-induced mice. Mice were pretreated with control shRNA or Atf6 shRNA for 6 weeks, then they were injected with olive oil or $\mathrm{CCl}_{4}$ for $24 \mathrm{~h}(n=12)$ : (a) the enzymatic rate method demonstrating the changes of serum ALT levels in the control group (control shRNA + olive oil), the Atf6 shRNA group (Atf6 shRNA + olive oil), the $\mathrm{CCl}_{4}$ group (control shRNA $+\mathrm{CCl}_{4}$ ), and the Atf6 shRNA $+\mathrm{CCl}_{4}$ group; (b) serum TBil levels measured using the diazonium method in the different experimental groups; (c) H\&E staining representing pathological changes in liver tissue and bar charts representing the proportion of necrotic liver tissue area; (d) western blot analysis of intrahepatic ATF6, RIP3, and p-MLKL expression among the different experimental groups; (e) qPCR analysis demonstrating the relative ATF6 and RIP3 expression among the

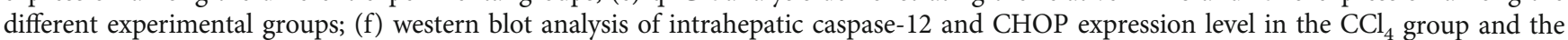
Atf6 shRNA $+\mathrm{CCl}_{4}$ group. ${ }^{* *} p<0.01$ versus the control shRNA group or the control shRNA $+\mathrm{CCl}_{4}$ group.

BALB/c mice were transduced with control shRNA or Atf6 shRNA for 6 weeks, then injected with olive oil or $\mathrm{CCl}_{4}$, and tissues were extracted after $24 \mathrm{~h}$. Compared with the control group (control shRNA + olive oil), Atf6 shRNA significantly increased the levels of serum ALT $(p<0.01$; Figure 5(a)) and TBil $(p<0.01$; Figure 5(b)); reduced the expression of ATF6 and RIP3 proteins; but increased MLKL phosphorylation $(p<0.01$; Figure $5(\mathrm{~d}))$. In the Atf6 shRNA $+\mathrm{CCl}_{4}$ group, Atf6 shRNA further increased the levels of serum ALT $(p<0.01$; Figure 5(a)), TBil $(p<0.01$; Figure 5(b)), and hepatocyte necrosis $(p<0.01$;
Figure 5(c)) and reduced the expression of ATF6 and RIP3 proteins; however, it increased MLKL phosphorylation compared with the control shRNA $+\mathrm{CCl}_{4}$ group $(p<0.01$; Figure $5(\mathrm{~d})$ ). In addition, Atf6 shRNA reduced the expression of Atf6 mRNA and Rip3 mRNA in the liver $(p<0.01$; Figure 5(e)), but it significantly increased $\mathrm{CCl}_{4}$-induced $\mathrm{CHOP}$ and caspase-12 protein expression in mouse livers $(p<0.01$; Figure 5(f)).

3.6. Rip3 Silencing Mitigates Liver Injury and ER Stress in $\mathrm{CCl}_{4}$-Induced Mice. Mice were transduced with control 


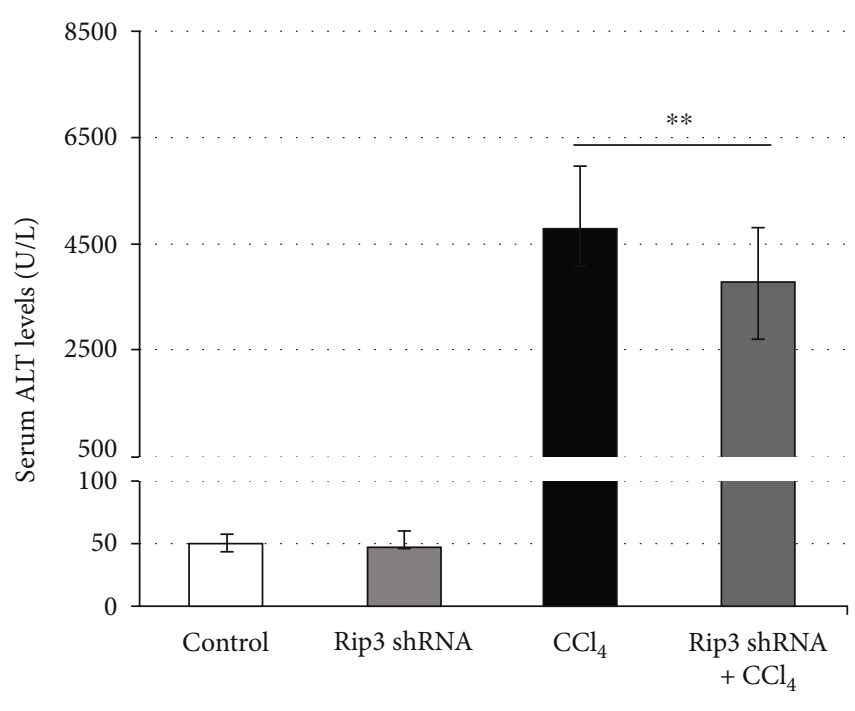

(a)
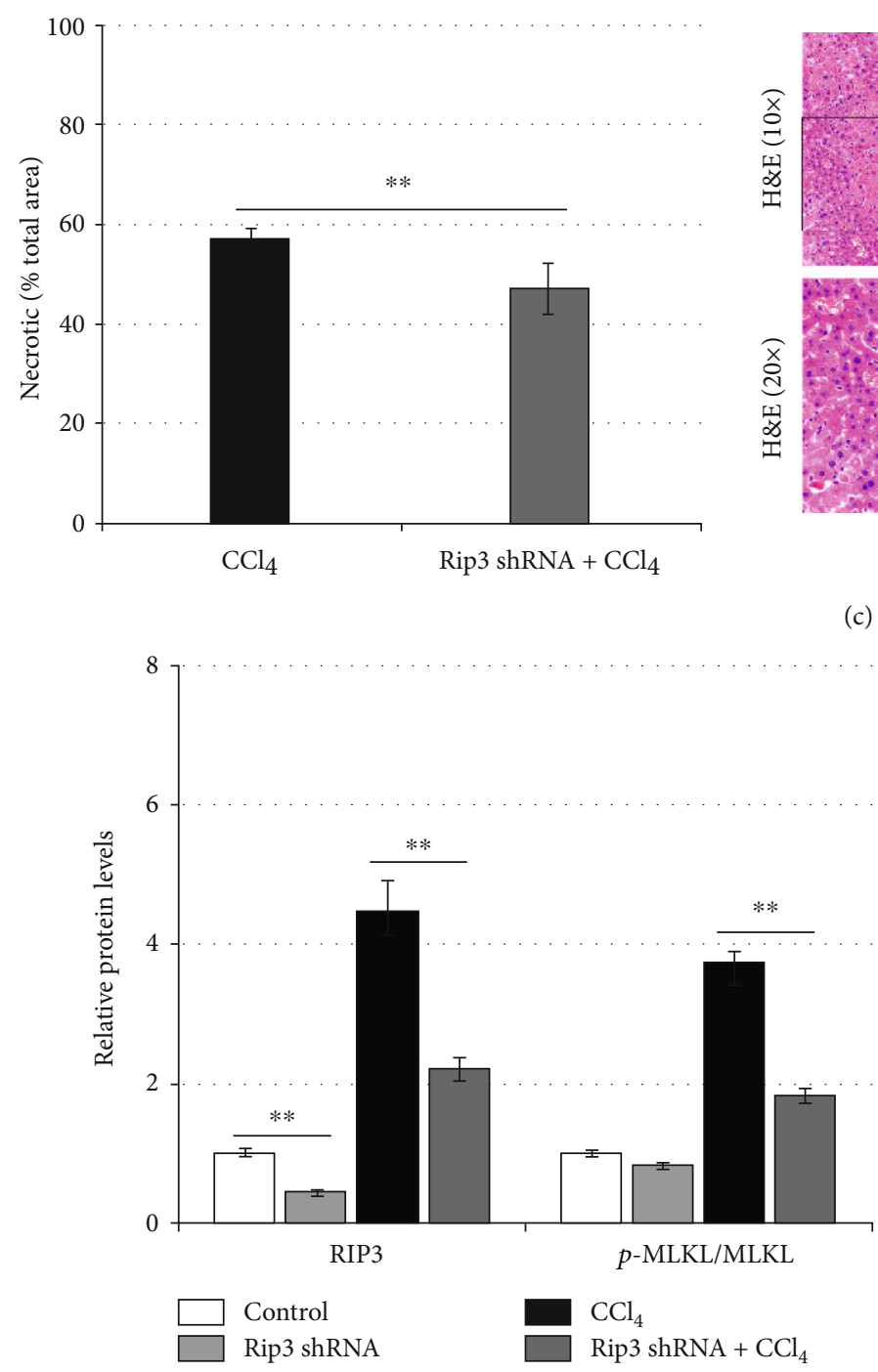

(c)

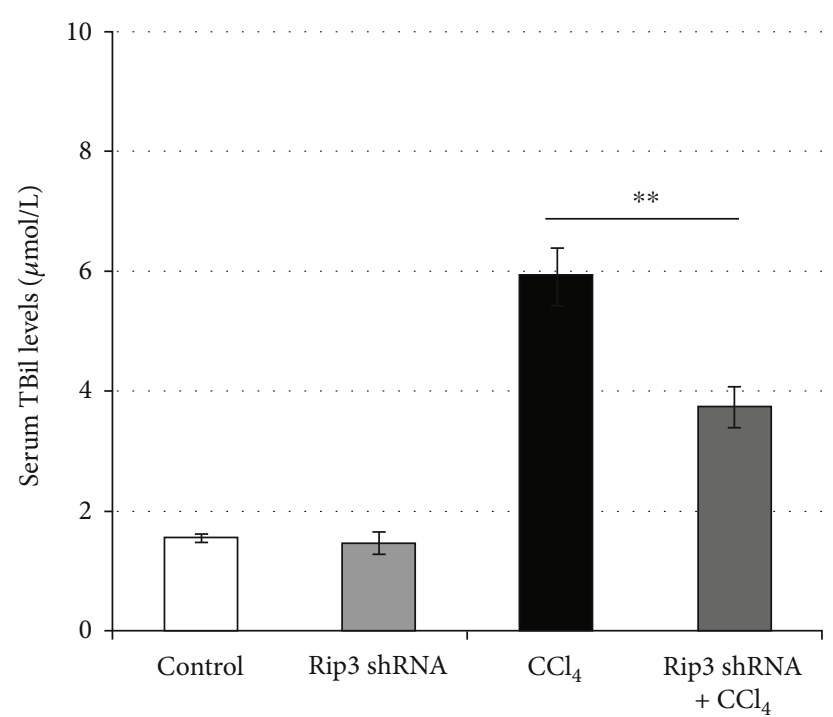

(b)
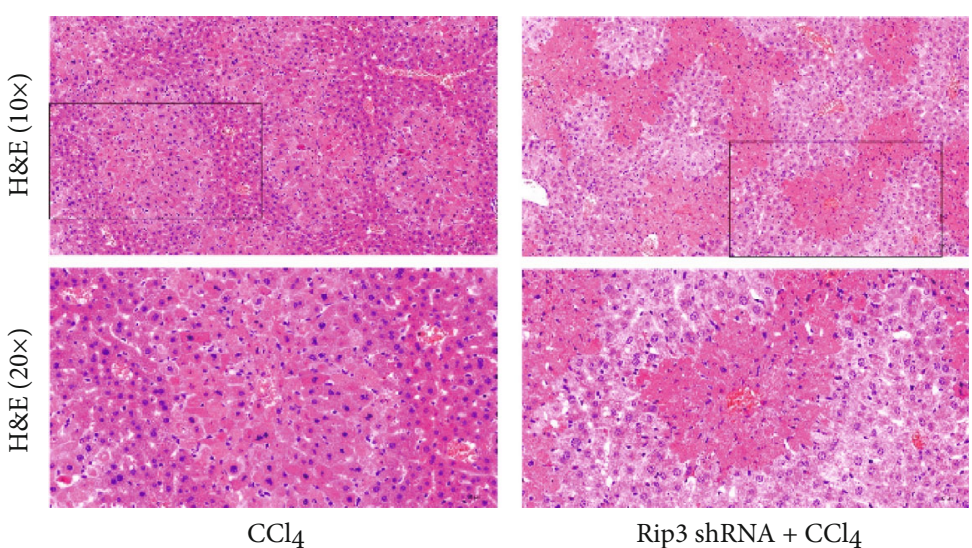

Rip3 shRNA + $\mathrm{CCl}_{4}$

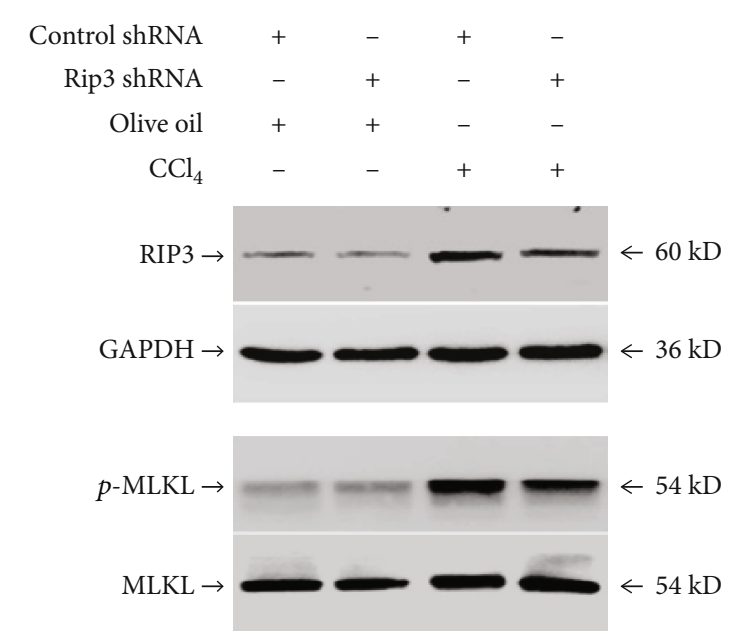

(d)

Figure 6: Continued. 


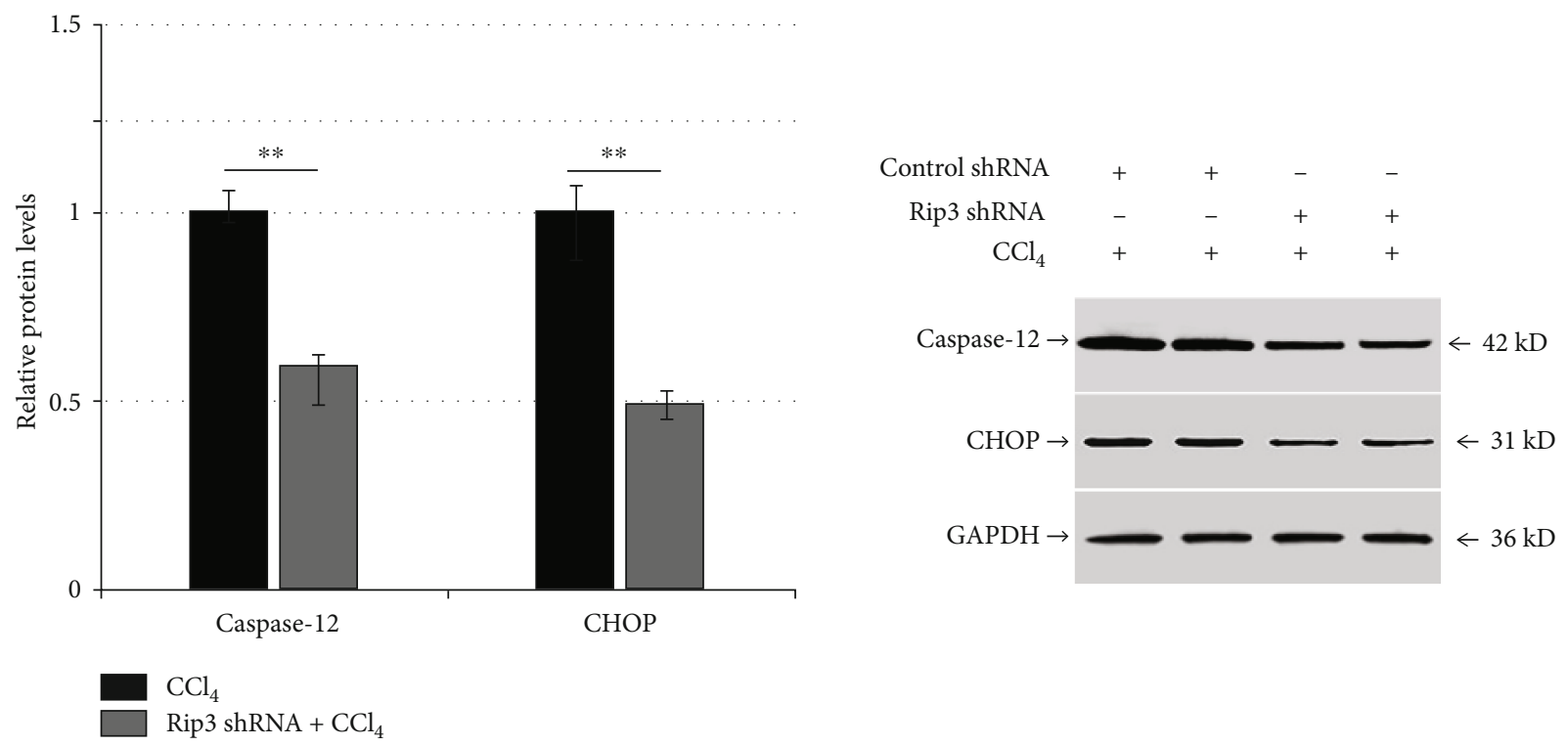

(e)

FIGURE 6: RIP3 downregulation mitigates liver injury and ER stress in $\mathrm{CCl}_{4}$-induced mice. Mice were administered the control shRNA or Rip3 shRNA, then they were injected with olive oil or $\mathrm{CCl}_{4}$ for another $24 \mathrm{~h}(n=12)$ : (a) the enzymatic rate method to detect the changes of serum ALT levels in the control group (control shRNA + olive oil), the Rip3 shRNA group (Rip3 shRNA + olive oil), the CCl 4 group (control shRNA $+\mathrm{CCl}_{4}$ ), and the Rip3 shRNA $+\mathrm{CCl}_{4}$ group; (b) serum TBil levels were measured using the diazonium method among the different experimental groups; (c) H\&E staining representing pathological changes in liver tissue and bar charts representing the proportion of necrotic liver tissue area; (d) western blotting examining the intrahepatic RIP3, and p-MLKL expression among the different

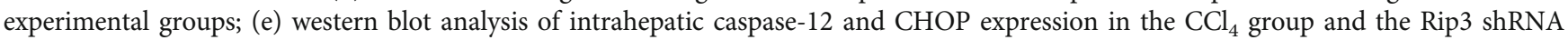
$+\mathrm{CCl}_{4}$ group. ${ }^{* *} p<0.01$ versus the control group or the control shRNA $+\mathrm{CCl}_{4}$ group.

shRNA or Rip3 shRNA for 6 weeks, then they were injected with olive oil or $\mathrm{CCl}_{4}$ for $24 \mathrm{~h}$. Compared with the control shRNA, Rip3 shRNA did not alter the serum levels of ALT $(p>0.05$; Figure $6(\mathrm{a}))$ or TBil $(p>0.05$; Figure $6(\mathrm{~b}))$. In addition, there were no significant changes in hepatocyte necrosis $(p>0.05$; data not shown) and intrahepatic pMLKL expression $(p>0.05$; Figure $6(\mathrm{~d}))$. Following $\mathrm{CCl}_{4}$ injection, Rip3 shRNA reduced the $\mathrm{CCl}_{4}$-induced serum ALT $(p<0.01)$ and TBil levels $(p<0.01)$ and hepatocyte necrosis $(p<0.01$; Figures 6(a)-6(c)). In addition, Rip3 shRNA reduced the expression of RIP3 and $\mathrm{p}-\mathrm{MLKL}$ protein in mouse liver compared with the control shRNA $+\mathrm{CCl}_{4}$ group $(p<0.01$; Figure $6(d))$. Similarly, Rip3 shRNA significantly reduced the intrahepatic expression of caspase-12 and $\mathrm{CHOP}$ protein in $\mathrm{CCl}_{4}$-induced mice $(p<0.01$; Figure 6(e)).

\section{Discussion}

The impact of silencing ATF6 and RIP3 on hepatocyte necroptosis in a hepatocyte model of ER stress and a mouse model of acute liver injury was examined. The results demonstrated that the incubation of LO2 cells with TG induced ER stress and necroptosis and upregulated RIP3 expression. ATF6 downregulation aggravated hepatocyte necroptosis and ER stress and reduced RIP3 expression in TG-induced LO2 cells. On the other hand, the downregulation of RIP3 reduced TG-induced hepatocyte necrosis and $\mathrm{p}-\mathrm{MLKL}$ and CHOP expression. Comparable results were observed in vivo; ATF6 and RIP3 expression was upregulated along with hepatocyte necroptosis following $\mathrm{CCl}_{4}$ or TM induction. Similarly, Atf6 downregulation aggravated liver injury, hepatocyte necroptosis, and ER stress and reduced RIP3 expression in $\mathrm{CCl}_{4}$-induced mice. However, Rip3 downregulation mitigated liver injury, hepatocyte necroptosis, and ER stress. Taken together, the results imply that ER stress could mediate hepatocyte necroptosis in acute liver injury. Upregulated ATF6 alleviated hepatocyte necroptosis and increased RIP3 expression during ER stress. However, ATF6 and RIP3 differentially control necroptosis in acute liver injury. The downregulation of RIP3 partially increased the protective effects of ATF6 on liver injury. Therefore, targeting RIP3 could be a potential treatment strategy for liver injury.

Controlling hepatocyte necroptosis to treat liver diseases is receiving increased attention [39]. ER stress-mediated necroptosis is independent of the TNFR1 pathway [40]. However, the crosstalk between ER stress and necroptosis and its significance remain unknown. The efficacy of the experimental models, for example, using $\mathrm{CCl}_{4}$ and $\mathrm{TM}$ to induce acute liver injury in mice and the TG-induced ER stress in LO2 cells, was validated in previous reports, and it was previously verified that ER stress mediates necroptosis [30, 41-43]. $\mathrm{CCl}_{4}$ is a well-documented hepatotoxin that induces acute liver injury by oxidative damage via its free radical metabolites [44-46]. TM can impede the glycosylation modification of newly synthesized proteins; therefore, it can cause damage to the ER function and induce ER stress in vivo and in vitro $[47,48]$. In agreement, $\mathrm{CCl}_{4}$ induced $\mathrm{ER}$ 


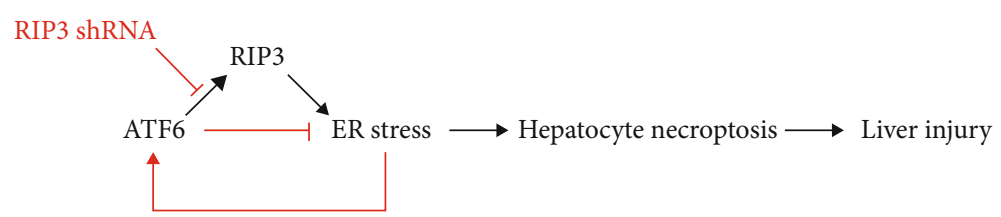

FIGURE 7: RIP3 shRNA reduces hepatocyte necroptosis. ATF6 plays multiple roles in acute liver injury and plays a dominant role in protecting the liver. It reduces hepatocyte necroptosis through a negative feedback regulation of ER stress. It also upregulates RIP3, which is not favorable to the recovery process. On the other hand, downregulating RIP3 reduces hepatocyte necroptosis by promoting the alleviation of ER stress.

stress and upregulated the expression of p-MLKL. Similarly, the ER stress inducers TM and TG induced p-MLKL expression in vivo and in vitro, respectively.

ATF6 is critical for the adaptive response of ER stress, and it is associated with the pathogenesis of various liver conditions [49]. For instance, the loss of ATF6 exacerbates liver steatosis that is caused by acute stress [50]. ATF6 overexpression improves insulin signal transduction and metabolic balance and slows down fatty liver degeneration in obese mice [51]. In addition, ATF6 knock-out mice challenged with TM exhibited sustained CHOP expression and increased liver steatosis [52-54]. Following cardiac ischemia-reperfusion injury, the knock-in of ATF6 reduced necrosis and apoptosis and improved cardiac function [55]. In our study, ATF6 knockdown increased the TG-induced p-MLKL expression and aggravated ER stress in LO2 cells. However, knockdown of ATF6 in $\mathrm{CCl}_{4}$-induced mice increased the phosphorylation of MLKL, aggravated ER stress, and aggravated liver injury. In combination, this suggested that ATF6 reduces necroptosis in hepatocytes by mitigating ER stress during acute liver injury. In addition, previous studies have reported that ATF6 ameliorates ER stress-mediated liver injury by upregulating sestrin 2 [56].

RIP3, which is a downstream kinase of RIP1, regulates the necroptosis signaling pathway. Phosphorylation of RIP3 at the Ser227 site is the key to its activation. This promotes the recruitment and activation of MLKL, leading to MLKL phosphorylation at Thr357 and Ser358, and therefore, initiating necroptosis signaling [57]. In addition, RIP3 directly phosphorylates RIP1 that further promotes necroptosis [58]. In ischemia-reperfusion injury, RIP3 was the downstream signal for ER stress in myocardial cells and its upregulation eventually leads to necroptosis [59]. The upregulation of RIP3 in chronic alcoholic liver injury leads to necroptosis and steatosis of hepatocytes. In contrast, the downregulation of RIP3 reduces liver injury and liver steatosis [60]. In patients with primary cholestasis, the expression of RIP3 and MLKL is upregulated. Further, RIP3 and MLKL expression is positively correlated with liver injury in mice with bile duct ligation. Knock-out of Rip3 improves necroptosis associated with cholestasis in mice [61]. In this study, the knockdown of Atf6 downregulated the expression of RIP3. Of interest, the knockdown of Rip3 decreased pMLKL expression and ER stress in $\mathrm{CCl}_{4}$-treated mice, which suggested that RIP3 and ATF6 might have different regulatory effects on necroptosis of hepatocytes in acute liver injury. In agreement with previous reports, RIP3 knockdown attenuated liver injury [62]. In addition, previous studies reported that RIP3 regulates ER stress and that knocking down RIP3 has a protective effect on cell damage $[63,64]$. There have been similar reports in acute myocardial infarction. Here, ZYZ-803, which can release $\mathrm{H}_{2} \mathrm{~S}$ and $\mathrm{NO}$ slowly, reduced ER stress-related necroptosis by downregulating the RIP3-CaMKII $\left(\mathrm{Ca}^{2+}\right.$-calmodulin-dependent protein kinase) signaling, instead of the classical "RIP1-RIP3MLKL" axis [65].

Based on results from our research, in the liver, the targeted knockdown of ATF6 reduced RIP3 and aggravated ER stress, and the knockdown of RIP3 reduced ER stress. The effect of RIP3 on ER stress in liver injury appears to be contradictory. However, our results suggested the following: (1) the activation of ATF6 is beneficial in reducing ER stress in acute liver injury; (2) hepatocyte ATF6 upregulated the expression of RIP3; and (3) knockdown of hepatocyte RIP3 promoted the relief of ER stress. However, ATF6 knockdown leads to a decrease in RIP3 expression and aggravates ER stress. ATF6 could have multiple roles in acute liver injury and could have a crucial role in protecting the liver. It reduces hepatocyte necroptosis through the negative feedback regulation of ER stress. In addition, ATF6 upregulates RIP3, which does not help the recovery process. Therefore, the knockdown of RIP3 reduced hepatocyte necroptosis and ER stress (Figure 7). In addition, previous research has shown that ATF6 has a protective and pathological role in certain liver disease models. For example, the loss of ATF6 can prevent steatosis that is caused by chronic ER stress; however, it can enhance steatosis that is caused by acute ER stress [66]. Other researchers observed that ATF6 plays a pathological role, ATF6 prevents alcohol-induced liver steatosis, and ATF6 overexpression induces steatosis in an SREBP-independent manner in a zebrafish alcoholic liver disease model [67]. In combination, AFT6 could have a protective and pathological role in liver disease, which might be related to its role as an important transcription factor by upregulating a variety of downstream target molecules that have different functions.

\section{Conclusion}

ATF6 has multiple roles in acute liver injury. It reduces hepatocyte necroptosis in acute liver injury, which could be attributed to the reduction in ER stress. However, ATF6 upregulates the expression of RIP3, which is not beneficial to the recovery from liver injury. The knockdown of RIP3 reduces hepatocyte necroptosis by mitigating ER stress. Therefore, targeting RIP3 could be promising in the future. 


\section{Data Availability}

The data that support the findings of this study are available from the corresponding author upon reasonable request.

\section{Conflicts of Interest}

The authors declare no conflict of interest.

\section{Authors' Contributions}

Mei-Ying Huang and Dian-Wei Wan contributed equally to this work.

\section{Acknowledgments}

This study was supported in part by the National Natural Science Foundation of China (81560110) and the Science and Technology Planning Projects of Guizhou Province (QKH.ZC[2019] 2803, QKH.PTRC [2017]5733-013, and gzwjkj2020-1-041).

\section{References}

[1] M. S. D'Arcy, "Cell death: a review of the major forms of apoptosis, necrosis and autophagy," Cell Biology International, vol. 43 , no. 6 , pp. 582-592, 2019.

[2] W. K. Saeed, D. W. Jun, K. Jang, and D. H. Koh, "Necroptosis signaling in liver diseases: an update," Pharmacological Research, vol. 148, article 104439, 2019.

[3] L. Dara, Z. X. Liu, and N. Kaplowitz, "Questions and controversies: the role of necroptosis in liver disease," Cell Death Discovery, vol. 2, no. 1, article 16089, 2016.

[4] R. F. Schwabe and T. Luedde, "Apoptosis and necroptosis in the liver: a matter of life and death," Nature Reviews. Gastroenterology \& Hepatology, vol. 15, no. 12, pp. 738-752, 2018.

[5] L. Sun, H. Wang, Z. Wang et al., "Mixed lineage kinase domain-like protein mediates necrosis signaling downstream of RIP3 kinase," Cell, vol. 148, no. 1-2, pp. 213-227, 2012.

[6] Z. M. Liu, Q. X. Chen, Z. B. Chen et al., "RIP3 deficiency protects against traumatic brain injury (TBI) through suppressing oxidative stress, inflammation and apoptosis: dependent on AMPK pathway," Biochemical and Biophysical Research Communications, vol. 499, no. 2, pp. 112-119, 2018.

[7] W. Chen, Z. Zhou, L. Li et al., "Diverse sequence determinants control human and mouse receptor interacting protein 3 (RIP3) and mixed lineage kinase domain-like (MLKL) interaction in necroptotic signaling," The Journal of Biological Chemistry, vol. 288, no. 23, pp. 16247-16261, 2013.

[8] M. E. Choi, D. R. Price, S. W. Ryter, and A. M. K. Choi, "Necroptosis: a crucial pathogenic mediator of human disease," JCI Insight, vol. 4, no. 15, 2019.

[9] X. Wu, K. L. Poulsen, C. Sanz-Garcia et al., "MLKL-dependent signaling regulates autophagic flux in a murine model of nonalcohol-associated fatty liver and steatohepatitis," Journal of Hepatology, vol. 73, no. 3, pp. 616-627, 2020.

[10] L. Cao and W. Mu, "Necrostatin-1 and necroptosis inhibition: pathophysiology and therapeutic implications," Pharmacological Research, vol. 163, p. 105297, 2021.
[11] J. L. Maiers and H. Malhi, "Endoplasmic reticulum stress in metabolic liver diseases and hepatic fibrosis," Seminars in Liver Disease, vol. 39, no. 2, pp. 235-248, 2019.

[12] J. Lukas, J. Pospech, C. Oppermann et al., "Role of endoplasmic reticulum stress and protein misfolding in disorders of the liver and pancreas," Advances in Medical Sciences, vol. 64, no. 2, pp. 315-323, 2019.

[13] M. Bian, J. He, H. Jin et al., "Oroxylin A induces apoptosis of activated hepatic stellate cells through endoplasmic reticulum stress," Apoptosis, vol. 24, no. 11-12, pp. 905-920, 2019.

[14] E. Szegezdi, S. E. Logue, A. M. Gorman, and A. Samali, "Mediators of endoplasmic reticulum stress-induced apoptosis," EMBO Reports, vol. 7, no. 9, pp. 880-885, 2006.

[15] P. Walter and D. Ron, "The unfolded protein response: from stress pathway to homeostatic regulation," Science, vol. 334, no. 6059, pp. 1081-1086, 2011.

[16] A. Kapoor and A. J. Sanyal, "Endoplasmic reticulum stress and the unfolded protein response," Clinics in Liver Disease, vol. 13, no. 4, pp. 581-590, 2009.

[17] A. Hughes, A. E. Oxford, K. Tawara, C. L. Jorcyk, and J. T. Oxford, "Endoplasmic reticulum stress and unfolded protein response in cartilage pathophysiology; contributing factors to apoptosis and osteoarthritis," International Journal of Molecular Sciences, vol. 18, no. 3, p. 665, 2017.

[18] Q. Luo, D. Yang, Q. Qi et al., "Role of the death receptor and endoplasmic reticulum stress signaling pathways in polyphyllin I-regulated apoptosis of human hepatocellular carcinoma HepG2 cells," BioMed Research International, vol. 2018, Article ID 5241941, 11 pages, 2018.

[19] A. Kishino, K. Hayashi, M. Maeda et al., "Caspase- 8 regulates endoplasmic reticulum stress-induced necroptosis independent of the apoptosis pathway in auditory cells," International Journal of Molecular Sciences, vol. 20, no. 23, p. 5896, 2019.

[20] Y. M. Yoon, J. H. Lee, S. P. Yun et al., "Tauroursodeoxycholic acid reduces ER stress by regulating of Akt-dependent cellular prion protein," Scientific Reports, vol. 6, no. 1, article 39838, 2016.

[21] R. P. Junjappa, P. Patil, K. R. Bhattarai, H. R. Kim, and H. J. Chae, "IRE1 $\alpha$ implications in endoplasmic reticulum stressmediated development and pathogenesis of autoimmune diseases," Frontiers in Immunology, vol. 9, p. 1289, 2018.

[22] P. Hu, Z. Han, A. D. Couvillon, R. J. Kaufman, and J. H. Exton, "Autocrine tumor necrosis factor alpha links endoplasmic reticulum stress to the membrane death receptor pathway through IRElalpha-mediated NF-kappaB activation and down-regulation of TRAF2 expression," Molecular and Cellular Biology, vol. 26, no. 8, pp. 3071-3084, 2006.

[23] X. Lou, H. Zhu, L. Ning et al., "EZH2 regulates intestinal inflammation and necroptosis through the JNK signaling pathway in intestinal epithelial cells," Digestive Diseases and Sciences, vol. 64, no. 12, pp. 3518-3527, 2019.

[24] Y. Liao, Y. Yang, X. Wang, M. Wei, Q. Guo, and L. Zhao, "Oroxyloside ameliorates acetaminophen-induced hepatotoxicity by inhibiting JNK related apoptosis and necroptosis," Journal of Ethnopharmacology, vol. 258, article 112917, 2020.

[25] J. R. Jheng, K. S. Lau, Y. W. Lan, and J. T. Horng, “A novel role of ER stress signal transducer ATF6 in regulating enterovirus A71 viral protein stability," Journal of Biomedical Science, vol. 25, no. 1, p. 9, 2018.

[26] H. Liu, S. Xie, F. Fang, D. V. Kalvakolanu, and W. Xiao, "SHQ1 is an ER stress response gene that facilitates 
chemotherapeutics-induced apoptosis via sensitizing ERstress response," Cell Death \& Disease, vol. 11, no. 6, p. 445, 2020.

[27] M. J. Song and H. Malhi, "The unfolded protein response and hepatic lipid metabolism in non alcoholic fatty liver disease," Pharmacology \& Therapeutics, vol. 203, article 107401, 2019.

[28] J. Gautheron, M. Vucur, F. Reisinger et al., "A positive feedback loop between RIP3 and JNK controls non-alcoholic steatohepatitis," EMBO Molecular Medicine, vol. 6, no. 8, pp. 1062-1074, 2014.

[29] B. Lu, P. Zhang, M. Zhou et al., "Involvement of XBP1s in blue light-induced A2E-containing retinal pigment epithelium cell death," Ophthalmic Research, vol. 57, no. 4, pp. 252-262, 2017.

[30] R. D. Tian, Y. Q. Chen, Y. H. He et al., "Phosphorylation of eIF2 $\alpha$ mitigates endoplasmic reticulum stress and hepatocyte necroptosis in acute liver injury," Annals of Hepatology, vol. 19, no. 1, pp. 79-87, 2020.

[31] L. Wu, X. Huang, Y. Kuang, Z. Xing, X. Deng, and Z. Luo, "Thapsigargin induces apoptosis in adrenocortical carcinoma by activating endoplasmic reticulum stress and the JNK signaling pathway: an in vitro and in vivo study," Drug Design, Development and Therapy, vol. Volume 13, pp. 2787-2798, 2019.

[32] Y. Liu, X. Pan, S. Li et al., "Endoplasmic reticulum stress restrains hepatocyte growth factor expression in hepatic stellate cells and rat acute liver failure model," Chemico-Biological Interactions, vol. 277, pp. 43-54, 2017.

[33] R. C. Simmonds, "Bioethics and animal use in programs of research, teaching, and testing," in Management of Animal Care and Use Programs in Research, Education, and Testing, R. H. Weichbrod, T. GAH, and J. N. Norton, Eds., CRC Press, Boca Raton, FL, 2018.

[34] Y. J. Tang, H. Chen, Y. Yi et al., "Inhibition of eIF2 $\alpha$ dephosphorylation protects hepatocytes from apoptosis by alleviating ER stress in acute liver injury," BioMed Research International, vol. 2020, Article ID 2626090, 16 pages, 2020.

[35] W. G. Huang, J. Wang, Y. J. Liu et al., "Endoplasmic reticulum stress increases multidrug-resistance protein 2 expression and mitigates acute liver injury," Current Molecular Medicine, vol. 20, no. 7, pp. 548-557, 2020.

[36] G. P. Boivin, D. L. Hickman, M. A. Creamer-Hente, K. R. Pritchett-Corning, and N. A. Bratcher, "Review of $\mathrm{CO}_{2}$ as a euthanasia agent for laboratory rats and mice," Journal of the American Association for Laboratory Animal Science, vol. 56, no. 5, pp. 491-499, 2017.

[37] J. G. Kralj, M. S. Munson, and D. Ross, “Total protein quantitation using the bicinchoninic acid assay and gradient elution moving boundary electrophoresis," Electrophoresis, vol. 35, no. 12-13, pp. 1887-1892, 2014.

[38] G. Lippi, M. Dipalo, R. Musa et al., "Evaluation of the analytical performances of the novel Beckman Coulter AU5800," Clinical Biochemistry, vol. 45, no. 6, pp. 502-504, 2012.

[39] X. Li, G. Dong, H. Xiong, and H. Diao, "A narrative review of the role of necroptosis in liver disease: a double-edged sword," Ann Transl Med., vol. 9, no. 5, p. 422, 2021.

[40] S. Saveljeva, S. L. Mc Laughlin, P. Vandenabeele, A. Samali, and M. J. Bertrand, "Endoplasmic reticulum stress induces ligand-independent TNFR1-mediated necroptosis in L929 cells," Cell Death \& Disease, vol. 6, no. 1, article e1587, 2015.

[41] G. Chen, X. Yang, Y. He et al., "Inhibiting alpha subunit of eukaryotic initiation factor 2 dephosphorylation protects injured hepatocytes and reduces hepatocyte proliferation in acute liver injury," Croatian Medical Journal, vol. 60, no. 6, pp. 532-544, 2019.

[42] Y. He, J. Long, W. Zhong, Y. Fu, Y. Li, and S. Lin, "Sustained endoplasmic reticulum stress inhibits hepatocyte proliferation via downregulation of c-Met expression," Molecular and Cellular Biochemistry, vol. 389, no. 1-2, pp. 151-158, 2014.

[43] Y. Ren, L. Liu, Y. Li et al., "Development and validation of a scoring system to predict progression to acute-on-chronic liver failure in patients with acute exacerbation of chronic hepatitis B," Hepatology Research, vol. 48, no. 9, pp. 692-700, 2018.

[44] S. Y. Lin, X. Dan, X. X. du et al., "Protective effects of salidroside against carbon tetrachloride (CCl4)-induced liver injury by initiating mitochondria to resist oxidative stress in mice," International Journal of Molecular Sciences, vol. 20, no. 13, 2019.

[45] C. Dai, X. Xiao, D. Li et al., "Chloroquine ameliorates carbon tetrachloride-induced acute liver injury in mice via the concomitant inhibition of inflammation and induction of apoptosis," Cell Death \& Disease, vol. 9, no. 12, p. 1164, 2018.

[46] M. Boll, W. D. Lutz, E. Becker, and A. Stampfl, "Mechanism of carbon tetrachloride-induced hepatotoxicity. Hepatocellular damage by reactive carbon tetrachloride metabolites," Zeitschrift für Naturforschung C, vol. 56, no. 7-8, pp. 649-659, 2001.

[47] Y. Chen, H. Zhang, Y. Chen et al., "Resveratrol alleviates endoplasmic reticulum stress-associated hepatic steatosis and injury in mice challenged with tunicamycin," Molecular Nutrition \& Food Research, vol. 64, no. 14, article e2000105, 2020.

[48] B. Yan, S. Liu, X. Li, Y. Zhong, F. Tong, and S. Yang, "Preconditioning with endoplasmic reticulum stress alleviated heart ischemia/reperfusion injury via modulating IRE1/ATF6/RACK1/PERK and PGC- $1 \alpha$ in diabetes mellitus," Biomedicine \& Pharmacotherapy, vol. 118, article 109407, 2019.

[49] X. Liu and R. M. Green, "Endoplasmic reticulum stress and liver diseases," Liver Research, vol. 3, no. 1, pp. 55-64, 2019.

[50] D. DeZwaan-McCabe, R. D. Sheldon, M. C. Gorecki et al., "ER stress inhibits liver fatty acid oxidation while unmitigated stress leads to anorexia-induced lipolysis and both liver and kidney steatosis," Cell Reports, vol. 19, no. 9, pp. 1794-1806, 2017.

[51] X. Chen, F. Zhang, Q. Gong et al., "Hepatic ATF6 increases fatty acid oxidation to attenuate hepatic steatosis in mice through peroxisome proliferator-activated receptor $\alpha$," Diabetes, vol. 65, no. 7, pp. 1904-1915, 2016.

[52] M. H. Kim, T. B. Aydemir, J. Kim, and R. J. Cousins, "Hepatic ZIP14-mediated zinc transport is required for adaptation to endoplasmic reticulum stress," Proceedings of the National Academy of Sciences of the United States of America, vol. 114, no. 29, pp. E5805-E5814, 2017.

[53] K. Yamamoto, K. Takahara, S. Oyadomari et al., "Induction of liver steatosis and lipid droplet formation in ATF6alphaknockout mice burdened with pharmacological endoplasmic reticulum stress," Molecular Biology of the Cell, vol. 21, no. 17, pp. 2975-2986, 2010.

[54] X. Sun, W. Li, Y. Deng et al., "Hepatic conditional knockout of ATF6 exacerbates liver metabolic damage by repressing autophage through MTOR pathway," Biochemical and Biophysical Research Communications, vol. 505, no. 1, pp. 4550, 2018.

[55] R. B. Sharma, J. T. Snyder, and L. C. Alonso, "Atf6 $\alpha$ impacts cell number by influencing survival, death and proliferation," Molecular Metabolism, vol. 27S, pp. S69-S80, 2019. 
[56] K. H. Jegal, S. M. Park, S. S. Cho et al., “Activating transcription factor 6-dependent sestrin 2 induction ameliorates ER stress-mediated liver injury," Biochimica et Biophysica Acta (BBA) - Molecular Cell Research, vol. 1864, no. 7, pp. 12951307, 2017.

[57] X. M. Zhao, Z. Chen, J. B. Zhao et al., "Hsp90 modulates the stability of MLKL and is required for TNF-induced necroptosis," Cell Death \& Disease, vol. 7, no. 2, article e2089, 2016.

[58] Y. Zhang, X. Chen, C. Gueydan, and J. Han, "Plasma membrane changes during programmed cell deaths," Cell Research, vol. 28, no. 1, pp. 9-21, 2018.

[59] P. Zhu, S. Hu, Q. Jin et al., "Ripk3 promotes ER stress-induced necroptosis in cardiac IR injury: a mechanism involving calcium overload/XO/ROS/mPTP pathway," Redox Biology, vol. 16, pp. 157-168, 2018.

[60] S. Wang, H. M. Ni, K. Dorko et al., "Increased hepatic receptor interacting protein kinase 3 expression due to impaired proteasomal functions contributes to alcohol-induced steatosis and liver injury," Oncotarget, vol. 7, no. 14, pp. 17681-17698, 2016.

[61] M. B. Afonso, P. M. Rodrigues, A. L. Simão et al., "Activation of necroptosis in human and experimental cholestasis," Cell Death \& Disease, vol. 7, no. 9, article e2390, 2016.

[62] M. Zhao, L. Lu, S. Lei et al., "Inhibition of receptor interacting protein kinases attenuates cardiomyocyte hypertrophy induced by palmitic acid," Oxidative Medicine and Cellular Longevity, vol. 2016, Article ID 1451676, 13 pages, 2016.

[63] J. Zhong, Y. Tan, J. Lu et al., "Therapeutic contribution of melatonin to the treatment of septic cardiomyopathy: a novel mechanism linking Ripk3-modified mitochondrial performance and endoplasmic reticulum function," Redox Biology, vol. 26, article 101287, 2019.

[64] L. Ying, J. Benjanuwattra, S. C. Chattipakorn, and N. Chattipakorn, "The role of RIPK3-regulated cell death pathways and necroptosis in the pathogenesis of cardiac ischaemia-reperfusion injury," Acta Physiologica, vol. 231, no. 2 , article e13541, 2021

[65] L. Chang, Z. Wang, F. Ma et al., "ZYZ-803 mitigates endoplasmic reticulum stress-related necroptosis after acute myocardial infarction through downregulating the RIP3-CaMKII signaling pathway," Oxidative Medicine and Cellular Longevity, vol. 2019, Article ID 6173685, 18 pages, 2019.

[66] A. Cinaroglu, C. Gao, D. Imrie, and K. C. Sadler, “Activating transcription factor 6 plays protective and pathological roles in steatosis due to endoplasmic reticulum stress in zebrafish," Hepatology, vol. 54, no. 2, pp. 495-508, 2011.

[67] D. L. Howarth, C. Lindtner, A. M. Vacaru et al., "Activating transcription factor 6 is necessary and sufficient for alcoholic fatty liver disease in zebrafish," PLoS Genetics, vol. 10, no. 5, p. e1004335, 2017. 